\title{
ITWM
}

Fraunhofer

Institut

Techno- und

Wirtschaftsmathematik

J. Ohser, W. Nagel, K. Schladitz

The Euler number of discretized sets - on the choice of adjacency in homogeneous lattices 
(c) Fraunhofer-Institut für Techno- und

Wirtschaftsmathematik ITWM 2002

\section{ISSN 1434-9973}

Bericht 33 (2002)

Alle Rechte vorbehalten. Ohne ausdrückliche, schriftliche Genehmigung des Herausgebers ist es nicht gestattet, das Buch oder Teile daraus in irgendeiner Form durch Fotokopie, Mikrofilm oder andere Verfahren zu reproduzieren oder in eine für Maschinen, insbesondere Datenverarbeitungsanlagen, verwendbare Sprache zu übertragen. Dasselbe gilt für das Recht der öffentlichen Wiedergabe.

Warennamen werden ohne Gewährleistung der freien Verwendbarkeit benutzt.

Die Veröffentlichungen in der Berichtsreihe des Fraunhofer ITWM können bezogen werden über:

Fraunhofer-Institut für Techno- und

Wirtschaftsmathematik ITWM

Gottlieb-Daimler-Straße, Geb. 49

\section{Kaiserslautern}

Telefon: +49 (0) 6 31/2 05-32 42

Telefax: +49 (0) 6 31/2 05-4139

E-Mail: info@itwm.fhg.de

Internet: www.itwm.fhg.de 
Das Tätigkeitsfeld des Fraunhofer Instituts für Techno- und Wirtschaftsmathematik ITWM umfasst anwendungsnahe Grundlagenforschung, angewandte Forschung sowie Beratung und kundenspezifische Lösungen auf allen Gebieten, die für Techno- und Wirtschaftsmathematik bedeutsam sind.

In der Reihe »Berichte des Fraunhofer ITWM « soll die Arbeit des Instituts kontinuierlich einer interessierten Öffentlichkeit in Industrie, Wirtschaft und Wissenschaft vorgestellt werden. Durch die enge Verzahnung mit dem Fachbereich Mathematik der Universität Kaiserslautern sowie durch zahlreiche Kooperationen mit internationalen Institutionen und Hochschulen in den Bereichen Ausbildung und Forschung ist ein großes Potenzial für Forschungsberichte vorhanden. In die Berichtreihe sollen sowohl hervorragende Diplom- und Projektarbeiten und Dissertationen als auch Forschungsberichte der Institutsmitarbeiter und Institutsgäste zu aktuellen Fragen der Techno- und Wirtschaftsmathematik aufgenommen werden.

Darüberhinaus bietet die Reihe ein Forum für die Berichterstattung über die zahlreichen Kooperationsprojekte des Instituts mit Partnern aus Industrie und Wirtschaft.

Berichterstattung heißt hier Dokumentation darüber, wie aktuelle Ergebnisse aus mathematischer Forschungs- und Entwicklungsarbeit in industrielle Anwendungen und Softwareprodukte transferiert werden, und wie umgekehrt Probleme der Praxis neue interessante mathematische Fragestellungen generieren.

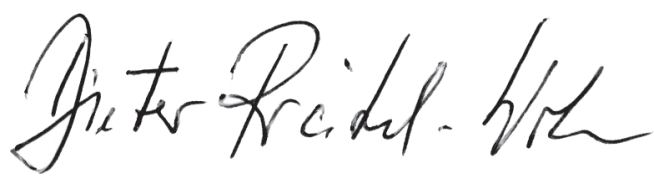

Prof. Dr. Dieter Prätzel-Wolters

Institutsleiter

Kaiserslautern, im Juni 2001 



\title{
The Euler number of discretized sets - on the choice of adjacency in homogeneous lattices
}

\author{
Joachim Ohser ${ }^{1}$, Werner Nagel ${ }^{2}$, and Katja Schladitz ${ }^{1}$ \\ February 21, 2002
}

\begin{abstract}
Two approaches for determining the Euler-Poincaré characteristic of a set observed on lattice points are considered in the context of image analysis - the integral geometric and the polyhedral approach. Information about the set is assumed to be available on lattice points only. In order to retain properties of the Euler number and to provide a good approximation of the true Euler number of the original set in the Euclidean space, the appropriate choice of adjacency in the lattice for the set and its background is crucial. Adjacencies are defined using tessellations of the whole space into polyhedrons. In $\mathbb{R}^{3}$, two new 14 adjacencies are introduced additionally to the well known 6 and 26 adjacencies. For the Euler number of a set and its complement, a consistency relation holds. Each of the pairs of adjacencies $(14.1,14.1),(14.2,14.2),(6,26)$, and $(26,6)$ is shown to be a pair of complementary adjacencies with respect to this relation. That is, the approximations of the Euler numbers are consistent if the set and its background (complement) are equipped with this pair of adjacencies. Furthermore, sufficient conditions for the correctness of the approximations of the Euler number are given. The analysis of selected microstructures and a simulation study illustrate how the estimated Euler number depends on the chosen adjacency. It also shows that there is not a uniquely best pair of adjacencies with respect to the estimation of the Euler number of a set in Euclidean space.
\end{abstract}

\section{Introduction}

In statistical physics and in materials' science the Euler number (or its density) is used as a characteristic describing the connectivity of the components (constituents) of a composite material or the pore space of a porous medium, see [18, 19] and [23].

From the mathematical point of view the Euler-Poincaré characteristic $\chi^{n}(X)$, or Euler number for short, of an $n$-dimensional set $X$ in the Euclidean space $\mathbb{R}^{n}$ is a basic quantity of integral geometry. By means of Crofton's intersection formulae, the quermassintegrals (Minkowski functionals or intrinsic volumes) can be expressed in terms of the Euler numbers $\chi^{k}$ defined on sections of $X$ with $k$-dimensional planes, $k=0, \ldots, n-1$, see [28]. This is a basis of the measurement of the quermassintegrals in image analysis. Definitions and properties of the Euler number are recalled in Section 2.

In the context of image analysis it is usually assumed that the set $X$ is observed on a point lattice $\mathbb{L}^{n}$. The intersection $X \cap \mathbb{L}^{n}$ is the mathematical expression for the observable

\footnotetext{
${ }^{1}$ Fraunhofer-Institut für Techno- und Wirtschaftsmathematik, Gottlieb-Daimler-Straße, 67663 Kaiserslautern

${ }^{2}$ Friedrich-Schiller-Universität Jena, Fakultät für Mathematik und Informatik, 07740 Jena
} 
information about $X$. In practical applications we consider the lattice $\mathbb{L}^{n}$ restricted to a bounded window $W \subset \mathbb{R}^{n}$. Let $1_{X}$ denote the characteristic function of $X$. The set $\left\{\left(x, 1_{X}(x)\right): x \in W \cap \mathbb{L}^{n}\right\}$ is said to be the (binary) image of $X$ observed in $W$, and the elements of the image are called pixels.

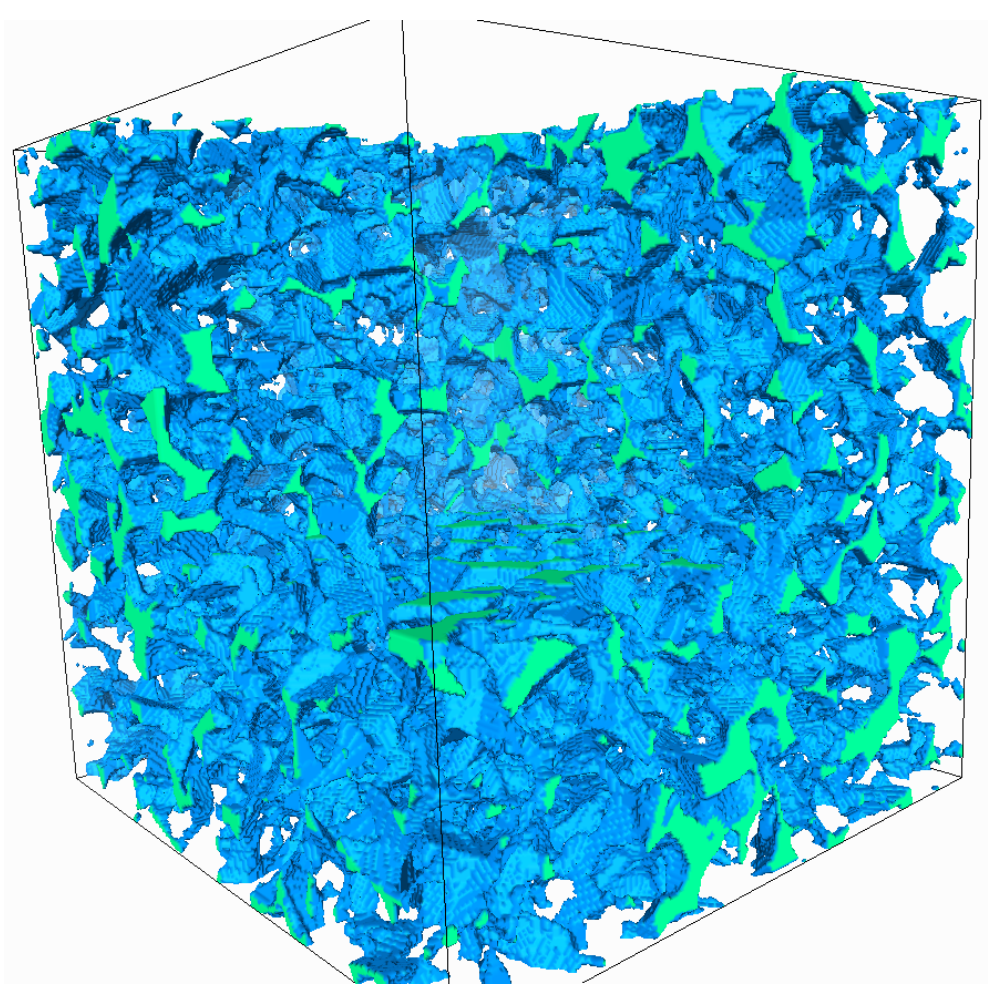

Figure 1: The microstructure of Fontainebleau sandstone. The XCT scan consists of $299 \times$ $300 \times 300$ pixels arranged in a simple cubic lattice of spacing $7.5 \mu \mathrm{m}$. This visualization of the $3 \mathrm{~d}$ image shows the pore space.

There are two ways of considering such discretized images. One way is based on theoretical foundation and development of formulae of discrete (lattice) geometry, in particular integral geometry on the lattice and introduction of discrete Minkowski functionals; see [31], [18] (or [19] for a short summary). In the present paper the alternative view is taken, namely that the analysis of the discretized image aims of a good estimation of parameters or features of the original (i.e. non-discretized) set in the Euclidean space. This aspect was introduced systematically in [30].

In order to reconstruct or approximate the connectivity of the set or to measure the Euler number, the crucial problem is the choice of the neighborhood graph which links lattice points of $\mathbb{L}^{n}$ in an appropriate way. Consistency of the pair of estimators for the Euler numbers of $X$ and of its complement $X^{c}$ can only be achieved if the neighborhoods for object (i.e. foreground) and background 'fit' according to Theorem 1 in Section 2. We call a pair of adjacencies complementary if it provides a pair of consistent estimators.

In $2 \mathrm{~d}$, there are the $(4,8),(8,4)$ and the $(6,6)$ adjacencies as pairs of complementary adjacencies. In this notation, the first component of a pair indicates the number of neighbors a lattice point is connected to if this lattice point belongs to $X$ (the object) and the second component denotes the number of used connections to neighbors if the lattice point belongs 
to the complement $X^{c}$ (the background). The $(6,6)$ adjacency is of particular importance since it is self-complementary in the sense that both object and background are treated the same way. For an introduction of these $2 \mathrm{~d}$ adjacencies see [30], [25], [23].

In $3 \mathrm{~d}$, the variety of reasonable pairs of adjacencies is much larger than in $2 \mathrm{~d}$ and not easy to survey. In image analysis, object and background are usually endowed with different neighborhoods in order to ensure a digital Jordan surface theorem. Typical pairs are $(6,26)$ and $(6,18)$, see e.g. $[11,14,16]$. In [12], the 18 neighborhood is modified to yield a 14 neighborhood. An exception to the asymmetry is [9], where both object and background are endowed with the same neighborhood but the neighborhood graph loses its invariance with respect to the translation group of the lattice.

Most algorithms for determining the Euler number, see [11, Section 8] and [16, Section 3.1.2] for surveys, work for 6 connectivity of the object and 26 connectivity of the background or vice versa. In [14], Lee, Poston, and Rosenfeld use algebraic topology to determine the contribution to the Euler number of all possible $2 \times 2 \times 2$ configurations ('marching cube algorithm'). The idea of this algorithm was published first by Serra, see [29]. Imiya and Eckhardt's [7] method is similar although with different theoretical background. Bieri and Nef [1] deduce a recursive algorithm from Hadwiger's recursive definition. This definition is the starting point for our integral geometric approach, too, see Subsection 3.2. Our polyhedral approach in Subsection 3.4 follows the setup suggested in [11, Section 8].

For $n>2$, the neighborhood graph does not describe uniquely the connectivity within the lattice cells. Thus, instead of neighborhood graphs, we use adjacency systems consisting of $k$ faces, $k=0, \ldots, n$. In Subsection 3.3, we reconsider the well known 6 and 26 adjacencies and introduce two new 14 adjacencies in $3 \mathrm{~d}$ using the new method of polyhedral tessellation of the lattice cells. The 14 adjacencies are self-complementary which is proved in 3.5. In Section 4 we show how the choice of adjacency influences the measurement results. Simulation studies show that using the 14 adjacencies can yield better results than $(6,26)$ or $(26,6)$ but that there are also models where they behave worse. This phenomenon is due to tiny structures in $X$ or in $X^{c}$, respectively, in the range of the lattice constant, i.e. in the range of the resolution of the equipment used for discretization.

In this paper we focus on proving results in the $3 \mathrm{~d}$ case. Basically, the approach for $n$-dimensional lattices is the same.

\section{The Euler number of a set and of its complement}

Assume that $X \subset \mathbb{R}^{n}$ is bounded and topologically closed, i.e. compact and that its Euler number $\chi^{n}(X)$ is defined. As an important subclass of such sets the reader may have in mind finite unions of compact convex sets (polyconvex sets). The set of all finite unions of compact convex sets is called the convex ring.

A set $X \subset \mathbb{R}^{n}$ is called topologically regular if $X=\overline{\left(X^{\text {int }}\right)}$, where $X^{\text {int }}$ and $\bar{X}$ denote the set of all interior points of $X$ and the closure of $X$, respectively. Topological regularity means that there are no isolated points or lower-dimensional parts (like fibers) in $X$. Notice that also the empty set $\emptyset$ is regular.

The complement $X^{c}=\mathbb{R}^{n} \backslash X$ of a bounded and topologically closed set $X$ is unbounded and topologically open. Furthermore, if $X$ belongs to the convex ring, neither the complement $X^{c}$ nor the topological closure of the complement $\overline{X^{c}}$ do belong to the convex ring. Hence it is not obvious how to introduce the Euler number of the complement and to establish relationships between $\chi^{n}(X)$ and $\chi^{n}\left(\overline{X^{c}}\right)$.

Finally, we recall the terms 'morphologically closed' and 'morphologically open' (see e.g. [30]): A set $X$ is said to be morphologically closed with respect to a compact set $B$ if 
$X \bullet B=X$ where $X \bullet B=(X \oplus \check{B}) \ominus B$ denotes the morphological closure of $X, X \oplus B$ and $X \ominus B$ are the (Minkowski) sum and the (Minkowski) difference, respectively, and $\breve{B}$ is the reflection of $B$. The set $X$ is said to be morphologically open with respect to $B$ if $X \circ B=X$ where $X \circ B=(X \ominus \check{B}) \oplus B$ is called the morphological opening. We call a set $X$ to be morphologically regular with respect to $B$ if it is morphologically open as well as morphologically closed with respect to $B$. Thus a morphologically regular set has no small (compared to $B$ ) particles, no thin spikes or lamellae, no small holes.

\subsection{Definition}

There are several equivalent ways for the definition of the Euler number of a set, see the books of Hadwiger [3], Matheron [17], Schneider [27], and Serra [30], cf. also [33].

In the case when $X$ belongs to the convex ring, i.e. it can be represented as a finite union $X=\cup_{i=1}^{m} X_{i}$ of compact convex sets $X_{i}$, the Euler number can be defined using the initial settings $\chi^{n}(\emptyset)=0$ and $\chi^{n}\left(X_{0}\right)=1$ for every compact convex set $X_{0} \neq \emptyset$ and the inclusion-exclusion principle, i.e.

$$
\begin{aligned}
& \chi^{n}(X)= \\
& \quad \sum_{i=1}^{m} \chi^{n}\left(X_{i}\right)-\sum_{i=1}^{m-1} \sum_{j=i+1}^{m} \chi^{n}\left(X_{i} \cap X_{j}\right)+\ldots+(-1)^{m+1} \chi^{n}\left(\bigcap_{i=1}^{m} X_{i}\right) .
\end{aligned}
$$

The inclusion-exclusion principle is equivalent to the additivity of the functional $\chi^{n}$, see [27].

For the Euler number of a polytope, i.e. the convex hull of a finite set of points, there is an alternative definition in terms of the numbers of its lower-dimensional faces. Let $P \subset \mathbb{R}^{n}$ be a polytope. For $k=0, \ldots, n$ denote by $\mathcal{F}^{k}(P)$ the set of all $k$-faces of $P$. In particular, $\mathcal{F}^{0}(P)$ is the set of vertices, $\mathcal{F}^{1}(P)$ the set of edges, $\mathcal{F}^{n-1}(P)$ the set of facets, and $\mathcal{F}^{n}(P)$ is the polytope itself, $\mathcal{F}^{n}(P)=P$. Furthermore, let $\# \mathcal{F}^{k}(P)$ be the cardinal number of $\mathcal{F}^{k}(P)$. Define the Euler number by

$$
\chi^{n}(P)=\sum_{k=0}^{n}(-1)^{k} \# \mathcal{F}^{k}(P)
$$

For a polytope, the Euler-Poincaré formula yields that the right hand side of (2) equals 1, see [32, Theorem 3.5.1]. Using (1), formula (2) can be additively extended to the case when $P$ is a finite union of polytopes, i.e. when $P$ is a polyhedron, see also [8, Section 4].

In the general case, when $X$ belongs to the convex ring, $\chi^{n}(X)$ can be equivalently introduced using Hadwiger's recursive definition. Let $\mathcal{L}_{k}^{n}$ be the set of all $k$-dimensional subspaces of $\mathbb{R}^{n}$. For $L \in \mathcal{L}_{k}^{n}$ denote by $L^{\perp}$ the orthogonal space. Consider a pair $\left(L_{x}, L_{x+\varepsilon u}\right)$ of parallel hyperplanes with distance $\varepsilon$ where $L \in \mathcal{L}_{n-1}^{n}, x \in L^{\perp}, u \in L^{\perp} \backslash\{o\}, \varepsilon>0$, and $L_{x}=L+x$. Starting with the initial setting $\chi^{0}(\emptyset)=0$ and $\chi^{0}(\{o\})=1$ in the zerodimensional case, the Euler number can be recursively defined for any dimension $n>0$ by

$$
\chi^{n}(X)=\sum_{x \in L^{\perp}} \lim _{\varepsilon \rightarrow 0}\left[\chi^{n-1}\left(X \cap L_{x}\right)-\chi^{n-1}\left(X \cap L_{x+\varepsilon u}\right)\right] .
$$

In (3), only finitely many items different from 0 occur. The limit differs from 0 when $L_{x}$ is an 'upper' tangent hyperplane to $X$ or a 'lower' tangent hyperplane to $X^{c}$. Notice that the value of the sum does not depend on the direction of the hyperplane. Here, the assumption that $X$ is a topologically closed set is crucial. Note that (3) can also be applied to unbounded sets whose complement is bounded. 
If $X$ belongs to the convex ring and both $X$ and $X^{c}$ are morphologically closed (i.e. $X$ is morphologically regular) with respect to a segment $[o, u]$ with $u \in L^{\perp} \backslash\{o\}$, then (3) can be rewritten as

$$
\begin{aligned}
\chi^{n}(X) & =\frac{1}{|u|} \int_{L^{\perp}}\left[\chi^{n-1}\left(X_{(x)} \cup X_{(x+u)}\right)-\chi^{n-1}\left(X_{(x)}\right)\right] d x \\
& =\frac{1}{|u|} \int_{L^{\perp}}\left[\chi^{n-1}\left(X_{(x)}\right)-\chi^{n-1}\left(X_{(x)} \cap X_{(x+u)}\right)\right] d x
\end{aligned}
$$

with $X_{(x)}=\left(X \cap L_{x}\right)_{-x}=X_{-x} \cap L \subset L$, see [24]. Roughly, the assumptions make sure that the details of the structure in $X$ and in $X^{c}$ are not too tiny compared with the structuring element $[o, u]$, i.e. with respect to the distance of the pair of sweeping hyperplanes used in (4) and (5). This guarantees that there is no doubt concerning the correct connectivities in the set $X$. Equations (4) and (5) can be used to prove the correctness of approximations of the Euler number based on local observations of discretized sets, see [22].

\subsection{Consistency relation}

In the $2 \mathrm{~d}$ case, a geometric interpretation of the Euler number of a set is $\chi^{2}=n_{0}-n_{1}$, where $n_{0}$ denotes the number of connected subsets ('components') and $n_{1}$ is the 'number of holes'. For the $3 \mathrm{~d}$ case it is $\chi^{3}=n_{0}+n_{1}-g$, where $n_{0}$ is again the number of connected components, and $n_{1}$ is the number of 'cavities'. Here $g$ is the genus of $X$. Heuristically, the genus is the maximal number of non-separating cuts through the set not increasing the number of connected components. Here a 'cut' must fully penetrate the set, see [16]. Hence, the genus of a set is the same as the genus of the complement. We remark that the terms '1st Betti number', 'number of handles', and 'number of tunnels' are used synonymously for 'genus'. For a more thorough discussion of Betti numbers see [15].

It is easy to see that for $2 \mathrm{~d}$ sets the Euler number is equal to the negative Euler number of the complement, and for the $3 \mathrm{~d}$ case the Euler numbers of a set and its complement are equal. In the following theorem, we prove this fact for arbitrary dimensions.

Theorem 1. (Consistency relation for the Euler number)

Assume that $X \subset \mathbb{R}^{n}, n \geq 1$, permits a representation $X=\cup_{i=1}^{m} X_{i}$, with compact convex sets $X_{i}$ such that all $X_{i}$ and all intersections $X_{i_{1}} \cap \ldots \cap X_{i_{k}}$ for $1 \leq i_{1}<\ldots<i_{k} \leq m$ and $k=2, \ldots, m$, are topologically regular. Then

$$
\chi^{n}\left(\overline{X^{c}}\right)=(-1)^{n+1} \chi^{n}(X) .
$$

Sketch of the proof: By induction over the dimension $n$ it can be shown easily that (6) is true for all compact convex sets $X$ with inner points (which implies topological regularity). Although $\overline{X^{c}}$ is an unbounded set which does not belong to the convex ring, the sum in (3) contains exactly one item different from zero. If $X=X_{1} \cup X_{2}$ where $X_{1}, X_{2}$ are compact convex sets and $X_{1}, X_{2}, X_{1} \cap X_{2}$ fulfill the regularity condition, the additivity of $\chi^{n}$ (the inclusion-exclusion principle) (1) provides

$$
\begin{aligned}
\chi^{n}\left(X_{1} \cup X_{2}\right) & =\chi^{n}\left(X_{1}\right)+\chi^{n}\left(X_{2}\right)-\chi^{n}\left(X_{1} \cap X_{2}\right) \\
& =(-1)^{n+1}\left[\chi^{n}\left(\overline{X_{1}^{c}}\right)+\chi^{n}\left(\overline{X_{2}^{c}}\right)-\chi^{n}\left(\overline{\left(X_{1} \cap X_{2}\right)^{c}}\right)\right] \\
& =(-1)^{n+1}\left[\chi^{n}\left(\overline{X_{1}^{c}}\right)+\chi^{n}\left(\overline{X_{2}^{c}}\right)-\chi^{n}\left(\overline{X_{1}^{c}} \cup \overline{X_{2}^{c}}\right)\right] \\
& =(-1)^{n+1} \chi^{n}\left(\overline{X_{1}^{c}} \cap \overline{X_{2}^{c}}\right) \\
& =(-1)^{n+1} \chi^{n}\left(\overline{\left(X_{1} \cup X_{2}\right)^{c}}\right) .
\end{aligned}
$$


Induction over the number $m$ of sets generating $X$ completes the proof.

Remark 1. The regularity condition for all intersections $X_{i_{1}} \cap \ldots \cap X_{i_{k}}$ means that these sets are either empty or have inner points, i.e., the sets $X_{1}, \ldots, X_{m}$ may not 'touch' each other in only one point or a set with a dimension less than $n$. It is obvious that without regularity conditions there is no chance to establish a relation between $\chi^{n}(X)$ and $\chi^{n}\left(\overline{X^{c}}\right)$. As an example let $X$ be a finite set of $m$ isolated points. Then $\chi^{n}(X)=m$ and $\chi^{n}\left(\overline{X^{c}}\right)=\chi^{n}(\emptyset)=0$.

An alternative proof of the consistency relation (6) can be given for those bounded sets $X$ for which both $X \cap B$ and $\overline{X^{c} \cap B}$ belong to the convex ring for all convex bodies $B$, see $\left[13\right.$, Appendix]. In this case the space $\mathbb{R}^{n}$ can be tessellated by convex bodies (e.g. cubes or parallelohedrons), and the additivity (1) can be applied. In particular, this approach works for bounded polyhedrons. We will use it for discretized sets.

\section{Approximation of the Euler number of a set}

\subsection{General}

The problem considered here is to find an appropriate approximation for the Euler number $\chi^{n}(X)$ based on the set $X \cap \mathbb{L}^{n}$ of lattice points. Formally, for every set $X$ find an approximation $\tilde{\chi}^{n}\left(X \cap \mathbb{L}^{n}\right)$ being close to the true value $\chi^{n}(X)$.

In order to describe approximation, we provide some concepts regarding homogeneous lattices. A subset $\mathbb{L}^{n}$ of the linear space $\mathbb{R}^{n}$ is called a homogeneous lattice if there exists a base $\left\{u_{1}, \ldots, u_{n}\right\}$ of $\mathbb{R}^{n}$ such that $\mathbb{L}^{n}=\left\{x \in \mathbb{R}^{n}: x=\sum_{i=1}^{n} \lambda_{i} u_{i}, \lambda_{i} \in \mathbb{Z}\right\}=U \mathbb{Z}^{n}$ with the matrix $U=\left(u_{1}, \ldots, u_{n}\right), \operatorname{det}(U) \neq 0$, where $\mathbb{Z}$ denotes the set of integers. The $\left|u_{i}\right|$ are the lattice spacings. Typical examples of homogeneous lattices are the cubic primitive lattice $\mathbb{L}^{n}=c_{0} \mathbb{Z}^{n}, c_{0}>0$, and the orthorhombic primitive lattice $\mathbb{L}^{n}=\operatorname{diag} \mathbb{Z}^{n}$ where diag is a diagonal matrix with non-zero entries. We remark that the homogeneous lattices - in their general setting - are known as the triclinic lattices. The unit cell $C$ of $\mathbb{L}^{n}$ is the Minkowski sum of the half-open segments $\left[o, u_{i}\right)=\left\{p u_{n}: 0 \leq p<1\right\}$ between the origin $o$ and the lattice points $u_{i} ; C=\left[o, u_{1}\right) \oplus \ldots \oplus\left[o, u_{n}\right)$. For more facts concerning lattices, see e.g. [2].

In this section, we present two approaches for the approximation of the Euler number. First, in Subsection 3.2, we recall the integral geometric approach based on a discretization of the recursive formulae (4) and (5).

Subsections 3.3 to 3.5 are devoted to the polyhedral approach. In image processing the standard solution for measuring the Euler number is to fix an adjacency relation for the lattice points and to determine the Euler number with respect to the corresponding polyhedron. Usually, the Euler number is computed by means of the Euler-Poincaré formula (2). This requires a clear and consistent definition of the $k$-faces to be counted. This definition is not unique for all neighborhood graphs or adjacency relations. Therefore, it is more precise to extend the neighborhood graph to the description of the whole adjacency system corresponding to a polyhedral approximation of the set $X$.

Very generally, an adjacency is a system of $k$-faces, $k=0, \ldots, n$. One way of obtaining an adjacency system is to use a tessellation $\mathcal{G}$ of $\mathbb{R}^{n}$ having the same translation group as the lattice. Then the adjacency system with respect to $\mathcal{G}$ - in the following denoted by $\mathbb{F}=\mathbb{F}(\mathcal{G})$ - is the set of all $k$-faces, $k=0, \ldots, n$, of polytopes belonging to $\mathcal{G}$.

Denote by $\mathbb{F} \sqcap X$ the union of all $k$-faces of $\mathcal{G}$ 'restricted' to $X, k=0, \ldots, n$ (i.e. the set of all $k$-faces of $\mathbb{F}$ for which all vertices belong to $X$; see also Section 3.4). This will be understood as a discretization of $X$. In Subsection 3.4 the following scheme is suggested:

$$
X \rightarrow X \cap \mathbb{L}^{n} \stackrel{\mathbb{F}}{\longrightarrow} \mathbb{F} \sqcap X \stackrel{(2)}{\longrightarrow} \tilde{\chi}^{n}(\mathbb{F} \sqcap X) \approx \chi^{n}(X) .
$$


For the complement $X^{c}$ we chose another adjacency system $\mathbb{F}_{c}$, and an approximation of the Euler number of $X^{c}$ can be obtained analogously to the above scheme from

$$
X^{c} \rightarrow X^{c} \cap \mathbb{L}^{n} \stackrel{\mathbb{F}_{c}}{\longrightarrow} \mathbb{F}_{c} \sqcap X^{c} \stackrel{(2)}{\longrightarrow} \tilde{\chi}^{n}\left(\mathbb{F}_{c} \sqcap X^{c}\right) \approx \chi^{n}\left(\overline{X^{c}}\right) .
$$

It is natural to use those pairs $\left(\mathbb{F}, \mathbb{F}_{c}\right)$ of adjacency systems for which the consistency relation (6) in the form $\tilde{\chi}^{n}\left(\mathbb{F}_{c} \sqcap X^{c}\right)=(-1)^{n+1} \tilde{\chi}^{n}(\mathbb{F} \sqcap X)$ is fulfilled for all $X$. Examples of such pairs are given in Subsection 3.5. Sufficient conditions for the correctness of the approximations are given in Subsection 3.6.

\subsection{The integral geometric approach}

In [22] an approximation of the Euler number is given together with morphological regularity conditions on $X$ and $X^{c}$ which are a consequence of the regularity conditions for (4) and (5). To describe this approach more detailed, we introduce intersections of the lattice $\mathbb{L}^{n}$ with $j$-dimensional subspaces $L \in \mathcal{L}_{j}^{n}$. We consider only those subspaces $L$ for which the intersection $\mathbb{L}^{n} \cap L$ forms a $j$-dimensional sublattice $\mathbb{L}^{j}$. Let $\mathcal{L}_{j}^{n}\left(\mathbb{L}^{n}\right)$ denote the set of all homogeneous $j$-dimensional sublattices of $\mathbb{L}^{n}$. The lattice $\left(\mathbb{L}^{j}\right)^{\perp}$ is the set of all points $y \in L^{\perp}$ with the property that $L_{y} \cap \mathbb{L}^{n}$ is a lattice on $L_{y}$. We call $\left(\mathbb{L}^{j}\right)^{\perp}$ the lattice orthogonal to $\mathbb{L}^{j}$. Note that $\left(\mathbb{L}^{j}\right)^{\perp}$ is not necessarily a sublattice of $\mathbb{L}^{n}$.

Let $\mathbb{L}^{n-1} \in \mathcal{L}_{n-1}^{n}\left(\mathbb{L}^{n}\right)$ and let $v_{n}$ be the base of the orthogonal lattice $\left(\mathbb{L}^{n-1}\right)^{\perp}$. Define $X_{(i)}=\left(X-i v_{n}\right) \cap \mathbb{L}^{n-1}$, i.e. $X_{(i)}$ is a discretization of the intersection $\left(X-i v_{n}\right) \cap L$. Then from (4) and (5) we obtain

$$
\begin{aligned}
\tilde{\chi}^{n}\left(X \cap \mathbb{L}^{n}\right): & =\sum_{i \in \mathbb{Z}}\left[\tilde{\chi}^{n-1}\left(X_{(i)} \cup X_{(i+1)}\right)-\tilde{\chi}^{n-1}\left(X_{(i)}\right)\right] \\
& =\sum_{i \in \mathbb{Z}}\left[\tilde{\chi}^{n-1}\left(X_{(i)}\right)-\tilde{\chi}^{n-1}\left(X_{(i)} \cap X_{(i+1)}\right)\right] .
\end{aligned}
$$

Recursive application of one of these formulae for decreasing dimension of the considered spaces leads to an approximation $\tilde{\chi}^{n}\left(X \cap \mathbb{L}^{n}\right)$ of $\chi(X)$. Bieri and Nef's [1] recursive algorithm is deduced directly from (7).

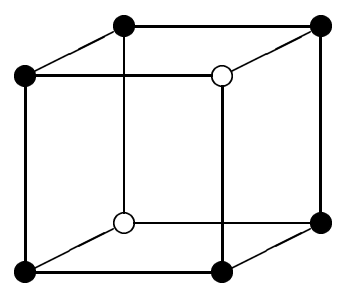

Figure 2: Pixel configuration in a $3 \mathrm{~d}$ image with a simple cubic lattice. The solid discs $\bullet$ are assigned to the pixels covered by $X$ whereas the circles $\circ$ are the pixels that hit the complement $X^{c}$. If $X$ is morphologically closed with respect to the edges and diagonals of the lattice cell, then $\chi(X)=1$ whereas (7) provides $\chi^{n}\left(X \cap \mathbb{L}^{n}\right)=0$. This difference is due to the fact that $X$ is not morphologically open with respect to space diagonals.

We recall from [22] the sufficient conditions for the correctness of the approximation. Let $C_{v}$ denote the unit cell of the lattice $\mathbb{L}_{v}^{n}$ with base $\left\{v_{1}, \ldots, v_{n}\right\}$ where $v_{n}, \ldots, v_{1}$ are the bases of the orthogonal lattices $\left(\mathbb{L}^{n-1}\right)^{\perp}, \ldots,\left(\mathbb{L}^{0}\right)^{\perp}$ used in the recursion. 
Theorem 2. Let $\mathcal{S}_{v}$ be the set of all closed segments that connect the vertices of the unit cell $C_{v} ; \mathcal{S}_{v}=\left\{[x, y]: x, y \in \mathcal{F}^{0}\left(\overline{C_{v}}\right), x \neq y,(x, y) \subset C_{v}\right\}$. If $X$ is morphologically regular with respect to all segments of $\mathcal{S}_{v}$ then $\tilde{\chi}^{n}\left(X \cap \mathbb{L}^{n}\right)$ provides the exact value of the Euler number, i.e. $\tilde{\chi}^{n}\left(X \cap \mathbb{L}^{n}\right)=\chi^{n}(X)$.

The proof follows from [22, Theorem 2], since $\mathbb{L}_{v}^{n}$ is by definition an orthorhombic primitive lattice.

If the regularity assumptions for $X$ are relaxed, then there may occur pixel configurations where the true value $\chi^{n}(X)$ differs from $\tilde{\chi}^{n}\left(X \cap \mathbb{L}^{n}\right)$ provided by (7) and (8), respectively, see Figure 2.

\subsection{Adjacencies, tessellations, and graphs}

For sets $X$ having a too tiny structure compared to the spacings of the observation lattice $\mathbb{L}^{n}$, i.e. sets not being morphologically regular with respect to the edges and diagonals of the cells of the lattice, one has to make guesses concerning the connectivity structure between the isolated lattice points. In image analysis, this guess is usually based on neighborhood graphs attached to the lattice.

We consider polyhedral approximations of the sets $X$ and $X^{c}$ rather than neighborhood graphs. These approximations are based on adjacencies. We first give a very general definition of adjacency:

Definition 1. An adjacency system $\mathbb{F}$ is a set of $k$-faces, $\mathbb{F}=\cup_{k=0}^{n} \mathcal{F}^{k}$ with

(i) each $k$-face $F \in \mathbb{F}$ is a subset of a (topologically closed) lattice cell and the vertices are lattice points

(ii) $\mathbb{F}$ is invariant with respect to lattice translations, $\mathbb{F}+x=\mathbb{F}$ for all $x \in \mathbb{L}^{n}$, and

(iii) the union of all polytopes of $\mathbb{F}$ is $\mathbb{R}^{n}$.

Adjacency systems can be constructed in various ways, for example using tessellations, collections or superpositions of tessellations, or forming the convex hull. Meaningful adjacencies are identified in Subsection 3.5 via the consistency relation (6). In this section we consider adjacency based on a special class of tessellations - so-called admissible tessellations.

A convex tessellation $\mathcal{G}$ of $\mathbb{R}^{n}$ is defined as a family $\left\{P_{1}, P_{2}, \ldots\right\}$ of polytopes (with inner

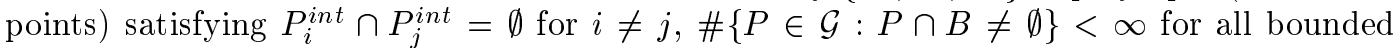
subsets $B \subset \mathbb{R}^{n}$, and $\cup_{i} P_{i}=\mathbb{R}^{n}$. In the following, we consider admissible tessellations only :

Definition 2. The tessellation $\mathcal{G}=\left\{P_{1}, P_{2}, \ldots\right\}$ is called an admissible tessellation with respect to the lattice $\mathbb{L}^{n}$ if

(i) the vertices of each tessellation cell are lattice points, i.e. $\mathcal{F}^{0}(P) \subset \mathbb{L}^{n}, P \in \mathcal{G}$,

(ii) the tessellation is invariant with respect to lattice translations, i.e. $\mathcal{G}+x=\mathcal{G}, x \in \mathbb{L}^{n}$,

(iii) the cells of the tessellation are subsets of the lattice cells, i.e., for every $P \in \mathcal{G}$ there exists a point $x \in \mathbb{L}^{n}$ such that $P \subseteq \bar{C}+x$, and

(iv) the intersection of two tessellation cells is either empty or a face of these cells, i.e., $P_{i} \cap P_{j}$ is an element of $\cup_{k=0}^{n-1}\left(\mathcal{F}^{k}\left(P_{i}\right) \cap \mathcal{F}^{k}\left(P_{j}\right)\right) \cup\{\emptyset\}, P_{i}, P_{j} \in \mathcal{G}, i \neq j$. 
To be more flexible, we consider also the superposition $\mathcal{G}$ of admissible tessellations $\mathcal{G}_{1}, \ldots, \mathcal{G}_{m}$ defined as

$$
\mathcal{G}=\left\{P: P=Q_{1} \cap \ldots \cap Q_{m}: Q_{1} \in \mathcal{G}_{1}, \ldots, Q_{m} \in \mathcal{G}_{m}\right\}, m \geq 1,
$$

see [20, Section 3.4]. Note that for $m>1$, the superposition $\mathcal{G}$ is not necessarily an admissible tessellation. In particular, there can exist vertices of cells of $\mathcal{G}$ that are not lattice points, $\mathcal{F}^{0}(\mathcal{G}) \supseteq \mathbb{L}^{n}$.

Now we introduce the systems $\mathcal{F}^{k}(\mathcal{G})$ of the $k$-faces of the superposition $\mathcal{G}=\left\{P_{1}, P_{2}, \ldots\right\}$ in the following way: $\mathcal{F}^{k}(\mathcal{G})=\cup_{i=1}^{\infty} \mathcal{F}^{k}\left(P_{i}\right), k=0, \ldots, n$. Obviously, admissible tessellations and their superpositions yield adjacencies.

Definition 3. Let $\mathcal{G}$ be the superposition of admissible tessellations. Then the set $\mathbb{F}(\mathcal{G})=$ $\cup_{k=0}^{n} \mathcal{F}^{k}(\mathcal{G})$ is called the adjacency system with respect to $\mathcal{G}$.

In order to link our polyhedral approach and the characterization of neighborhood usually used in image processing, we introduce the neighborhood graph $\Gamma$ consisting of non-oriented edges $\langle x, y\rangle$ that link lattice points $x, y \in \mathbb{L}^{n}$. Define the neighborhood relation for $\mathbb{F}$ as

$$
\gamma=\left\{\langle x, y\rangle: x, y \in \mathcal{F}^{0}(\bar{C}),(x, y) \subset C,[x, y] \subset \bigcup\left\{F \in \mathcal{F}^{1}(\mathbb{F})\right\}\right\} .
$$

Then the neighborhood graph with respect to $\mathbb{F}$ can be described by $\gamma ; \Gamma=\cup_{x \in \mathbb{L}^{n}}(\gamma+x)$. The connectivity $c=c(\Gamma)$ of the neighborhood graph is the order of the nodes; $c=\#\{\langle x, y\rangle \in$ $\left.\Gamma: y \in \mathbb{L}^{n}\right\}$ for every lattice point $x$. It is easy to see that $c=2 \# \gamma$. In the literature, adjacencies are usually called $c$ neighborhoods.

Consider the admissible tessellation $\mathcal{G}_{0}=\left\{\bar{C}+x: x \in \mathbb{L}^{n}\right\}$ consisting of the lattice cells. Then $\gamma_{0}=\left\{\left\langle o, u_{1}\right\rangle, \ldots,\left\langle o, u_{n}\right\rangle\right\}$ is the generator of the neighborhood graph $\Gamma_{0}$ of the adjacency $\mathbb{F}\left(\mathcal{G}_{0}\right)$, and the connectivity is $c\left(\Gamma_{0}\right)=2 n$. Since $c\left(\Gamma_{0}\right) \leq c(\Gamma)$ for all adjacencies $\mathbb{F}(\mathcal{G})$ with respect to an admissible tessellation $\mathcal{G}$ and with graph $\Gamma$, the adjacency $\mathbb{F}\left(\mathcal{G}_{0}\right)$ is said to be the minimal adjacency. The adjacency $\mathbb{F}\left(\mathcal{G}_{\max }\right)$ with respect to the superposition $\mathcal{G}_{\max }$ of the set of all admissible tessellations is called the maximum adjacency. Its neighborhood graph $\Gamma_{\max }$ is of order $c\left(\Gamma_{\max }\right)=3^{n}-1$.

Examples of 3d adjacencies. We consider the adjacencies in $3 \mathrm{~d}$ which correspond to the well known 6 and 26 neighborhoods. (For the 6 neighborhood each pixel is connected only by the edges of the lattice cells with its neighbors, and in the case of 26 neighborhood the pixels are connected by all edges, face diagonals and space diagonals of the cells.) Furthermore, we introduce two - apparently new - 14 neighborhoods. In order to describe the corresponding tessellations we introduce the vertices $x_{0}, \ldots, x_{7}$ of $C$ given by $x_{0}=o, x_{1}=u_{1}, x_{2}=u_{2}$, $x_{3}=u_{1}+u_{2}, x_{4}=u_{3}, x_{5}=u_{1}+u_{3}, x_{6}=u_{2}+u_{3}$, and $x_{7}=u_{1}+u_{2}+u_{3}$, where $\left\{u_{1}, u_{2}, u_{3}\right\}$ is the base of $\mathbb{L}^{3}$.

1. Clearly, for the $3 \mathrm{~d}$ case the minimal adjacency $\mathbb{F}\left(\mathcal{G}_{0}\right)$ corresponds to the 6 neighborhood; $c\left(\Gamma_{0}\right)=6$. The adjacency $\mathbb{F}\left(\mathcal{G}_{0}\right)$ is invariant with respect to rotation in the sense that $U M U^{-1} \mathbb{F}\left(\mathcal{G}_{0}\right)=\mathbb{F}\left(\mathcal{G}_{0}\right)$ for every rotation $M$ from the rotation group $\Omega\left(\mathbb{Z}^{3}\right)$ of the discrete space $\mathbb{Z}^{3}$. ( $\Omega\left(\mathbb{Z}^{3}\right)$ is called the octaeder group.)

2. Consider the tessellation of the unit cell into the 6 tetrahedrons $G_{1}, \ldots, G_{6}$ which are convex hulls of vertices of $C$ :

$$
\begin{array}{ll}
G_{1}=\operatorname{conv}\left(\left\{x_{0}, x_{1}, x_{3}, x_{7}\right\}\right), & G_{2}=\operatorname{conv}\left(\left\{x_{0}, x_{1}, x_{5}, x_{7}\right\}\right), \\
G_{3}=\operatorname{conv}\left(\left\{x_{0}, x_{2}, x_{3}, x_{7}\right\}\right), & G_{4}=\operatorname{conv}\left(\left\{x_{0}, x_{4}, x_{5}, x_{7}\right\}\right), \\
G_{5}=\operatorname{conv}\left(\left\{x_{0}, x_{4}, x_{6}, x_{7}\right\}\right), & G_{6}=\operatorname{conv}\left(\left\{x_{0}, x_{2}, x_{6}, x_{7}\right\}\right),
\end{array}
$$



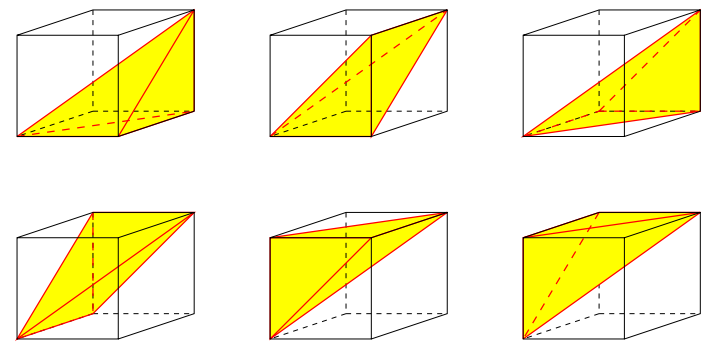

Figure 3: The tessellation of the unit cell defining the 14.1 neighborhood.

see Figure 3. Let $\mathcal{G}_{1}$ consist of all translations of the $G_{i}, \mathcal{G}_{1}=\left\{G_{i}+x: i=1, \ldots, 6, x \in\right.$ $\mathbb{L}^{3}$ \}. Then $\mathcal{G}_{1}$ is an admissible tessellation of $\mathbb{R}^{3}$ with respect to $\mathbb{L}^{3}$. For the corresponding adjacency $\mathbb{F}\left(\mathcal{G}_{1}\right)$, the set $\gamma$ consists of three edges of $C$, three face diagonals, and one space diagonal, i.e. $c(\Gamma)=14$. Hence $\mathbb{F}\left(\mathcal{G}_{1}\right)$ has a 14 neighborhood. Note that $\mathbb{F}\left(\mathcal{G}_{1}\right)$ is not invariant under rotation. But even besides its rotations there is a further adjacency based on a tessellation of $C$ into 6 tetrahedrons. To indicate the difference we call $\mathbb{F}_{14.1}=\mathbb{F}\left(\mathcal{G}_{1}\right)$ the 14.1 neighborhood.
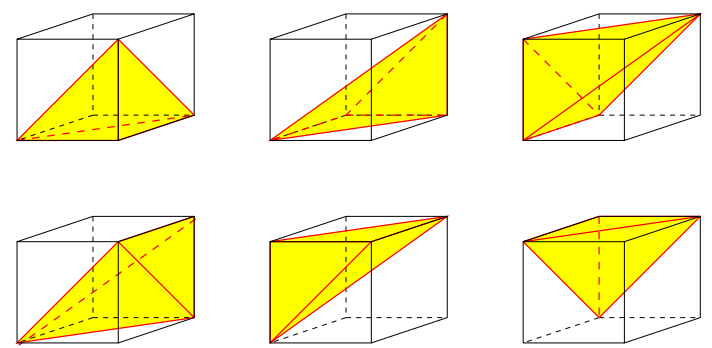

Figure 4: The tessellation of the unit cell defining the 14.2 neighborhood.

3. Consider now the tetrahedrons

$$
\begin{array}{ll}
G_{1}=\operatorname{conv}\left(\left\{x_{0}, x_{1}, x_{3}, x_{5}\right\}\right), & G_{2}=\operatorname{conv}\left(\left\{x_{0}, x_{2}, x_{3}, x_{7}\right\}\right), \\
G_{3}=\operatorname{conv}\left(\left\{x_{0}, x_{2}, x_{4}, x_{7}\right\}\right), & G_{4}=\operatorname{conv}\left(\left\{x_{0}, x_{3}, x_{5}, x_{7}\right\}\right), \\
G_{5}=\operatorname{conv}\left(\left\{x_{0}, x_{4}, x_{5}, x_{7}\right\}\right), & G_{6}=\operatorname{conv}\left(\left\{x_{2}, x_{4}, x_{6}, x_{7}\right\}\right)
\end{array}
$$

which tessellate the unit cell as shown in Figure 4. Again, the translations of the $G_{i}$ form an admissible tessellation $\mathcal{G}_{2}$, and the adjacency $\mathbb{F}_{14.2}=\mathbb{F}\left(\mathcal{G}_{2}\right)$ is a 14 neighborhood, too, in the following called the 14.2 neighborhood.

4. The neighborhood graph of the maximum adjacency is of order 26 . Hence we write $\mathbb{F}_{26}=\mathbb{F}\left(\mathcal{G}_{\max }\right)$. The maximum adjacency $\mathbb{F}_{26}$ is invariant with respect to rotation (as $\left.\mathbb{F}_{6}\right)$.

\subsection{The Euler number with respect to adjacency}

The elements of $\mathbb{F}(\mathcal{G})$ are the geometric objects to be used for the discretization of the set $X$ and for the computation of its Euler number.

For simplicity we first consider the case when $\mathbb{F}(\mathcal{G})$ is an adjacency with respect to an admissible tessellation. Then the set of the $k$-faces for which all vertices belong to $X$ can be formally introduced as $\mathcal{F}^{k}(\mathcal{G}) \sqcap X=\left\{F \in \mathcal{F}^{k}(\mathcal{G}): \mathcal{F}^{0}(F) \subseteq X\right\}$. 
If $\mathcal{G}$ is a superposition of admissible tessellations, there can occur difficulties with the extra vertices $\mathcal{F}^{0}(\mathcal{G}) \backslash \mathbb{L}^{n}$ in which the set $X$ is not observed. To overcome this problem, we assume that a vertex $z \in \mathcal{F}^{0}(\mathcal{G}) \backslash \mathbb{L}^{n}$ belongs to $X$ if there is an edge $\langle x, y\rangle$ of $\Gamma$ with $x, y \in X$ and $z \in[x, y]$. That is, $z$ lies on a segment between two neighboring nodes of the neighborhood graph $\Gamma$. In order to describe rigorously the discretization of $X$ with respect to $\mathbb{F}(\mathcal{G})$, we augment $X$ by the additional vertices:

$$
X^{*}=X \cup\left\{z \in \mathcal{F}^{0}(\mathcal{G}) \backslash \mathbb{L}^{n}: \text { there are } x, y \in X \text { with }\langle x, y\rangle \in \Gamma \text { and } z \in[x, y]\right\} .
$$

Now the discretization of the set $X$ with respect to $\mathbb{F}(\mathcal{G})$ can be defined as follows:

Definition 4. Let $\mathcal{F}^{k}(\mathcal{G}) \sqcap X$ denote the set of those $k$-faces for which all vertices belong to $X^{*}$ and

$$
\mathcal{F}^{k}(\mathcal{G}) \sqcap X=\left\{F \in \mathcal{F}^{k}(\mathcal{G}): \mathcal{F}^{0}(F) \subseteq X^{*}\right\}
$$

For a superposition $\mathcal{G}$ of admissible tessellations we call

$$
\mathbb{F}(\mathcal{G}) \sqcap X=\cup_{k=0}^{n} \mathcal{F}^{k}(\mathcal{G}) \sqcap X
$$

the discretization of $X$ with respect to the adjacency $\mathbb{F}(\mathcal{G})$.

Notice that if $X$ is a bounded subset of $\mathbb{R}^{n}$, its discretization is a polyhedron, i.e. a finite union of polytopes. Thus the Euler-Poincaré formula (3) yields an easy formula for the computation of the Euler number,

$$
\tilde{\chi}^{n}(\mathbb{F}(\mathcal{G}) \sqcap X)=\sum_{k=0}^{n}(-1)^{k} \#\left(\mathcal{F}^{k}(\mathcal{G}) \sqcap X\right),
$$

see also $[8,(4)]$.

For practical applications it is helpful to give also a 'local version' of this formula based on 'local versions' of the $\mathcal{F}^{k}(\mathcal{G}) \sqcap X$. The approximation $\tilde{\chi}^{n}(\mathbb{F}(\mathcal{G}) \sqcap X)$ can be based on estimates in each of the cells and their combination according to the inclusion-exclusion principle. In order to ensure that each $k$-face is counted once only, we have to apply some kind of edge correction. Here we choose weighting the $k$-faces with the reciprocal of the number of closed lattice cells they intersect.

For $\ell \geq k$ let $\mathcal{F}_{0}^{k \ell} \sqcap X$ be the set of all $k$-faces $F \in \mathcal{F}^{k}(\mathcal{G}) \sqcap X$ with:

(i) There is an $l$-face $F_{\ell} \in \mathcal{F}^{\ell}(\bar{C})$ such that $F \subseteq F_{\ell}$ and

(ii) there is no $j$-face $F_{j} \in \mathcal{F}^{j}(\bar{C}), j<\ell$, such that $F \subseteq F_{j}$.

Then (9) can be rewritten as

$$
\tilde{\chi}^{n}(\mathbb{F}(\mathcal{G}) \sqcap X)=\sum_{x \in \mathbb{L}^{n}} \sum_{k=0}^{n}(-1)^{k} \sum_{\ell=k}^{n} 2^{\ell-n} \#\left(\mathcal{F}_{0}^{k \ell} \sqcap X_{-x}\right) .
$$

For the $3 \mathrm{~d}$ case the interpretation of the weights is as follows: All vertices $\mathcal{F}_{0}^{0,0} \sqcap X$ get weight $1 / 8$. The edges $\mathcal{F}_{0}^{1,1} \sqcap X, \mathcal{F}_{0}^{1,2} \sqcap X$, and $\mathcal{F}_{0}^{1,3} \sqcap X$ get $1 / 4,1 / 2$, and 1 , respectively. The faces $\mathcal{F}_{0}^{2,2} \sqcap X$ and $\mathcal{F}_{0}^{2,3} \sqcap X$ are equipped with $1 / 2$ and 1 , respectively, and all cells $\mathcal{F}_{0}^{3,3} \sqcap X$ with 1 .

Remark 2. The local version (10) of (9) is easy to apply for $\mathbb{F}_{6}, \mathbb{F}_{14.1}$, and $\mathbb{F}_{14.2}$, but tedious 
for $\mathbb{F}_{26}$. For $\mathbb{F}_{26}$, the set $\mathcal{F}_{0}^{k \ell} \sqcap X_{-x}$ can be obtained from the convex hull of the vertices of $\bar{C}$ belonging to $X$.

Remark 3. Algorithmically, using the half open lattice cell $C$ is easier. Let $\mathcal{F}_{0}^{k} \sqcap X=$ $\left\{F \in \mathcal{F}^{k}(\mathcal{G}) \sqcap X: F \cap C \neq \emptyset, F \subseteq \bar{C}\right\}, \quad k=0, \ldots, n$. Then (9) can be rewritten as $\tilde{\chi}^{n}(\mathbb{F}(\mathcal{G}) \sqcap X)=\sum_{x \in \mathbb{L}^{n}} \sum_{k=0}^{n}(-1)^{k} \#\left(\mathcal{F}_{0}^{k} \sqcap X_{-x}\right)$.

For $n=3$ let $x_{0}, \ldots, x_{7}$ denote the vertices of the unit cell $C$ (defined as in the examples of Section 3.3). Following the technique described in [16], we define a function

$$
g(x)=\sum_{i=0}^{7} 2^{i} 1_{X}\left(x+x_{i}\right), \quad x \in \mathbb{L}^{3},
$$

with integer values $0, \ldots, 255$. This means that depending on whether the vertex $x_{i}$ hits the object or the background it gets the weight $2^{i}$ or 0 , respectively, see also Figure 5a). Furthermore, let $W \subset \mathbb{R}^{3}$ be a compact window with $X \oplus \bar{C} \subseteq W$. We introduce a vector $h$ with coefficients

$$
h_{i}=\sum_{x \in \mathbb{L}^{3} \cap W} \delta_{i}(g(x)), \quad i=0, \ldots, 255,
$$

where $\delta$ denotes Kronecker's delta, $\delta_{i}(j)=1$ for $i=j$ and $\delta_{i}(j)=0$ otherwise. Then (10) can be rewritten as

$$
\tilde{\chi}^{3}(\mathbb{F}(\mathcal{G}) \sqcap X)=\frac{1}{8} h w,
$$

where $w$ is given in Tables 1 and 2 for the adjacencies $\mathbb{F}_{6}, \mathbb{F}_{14.1}, \mathbb{F}_{14.2}$, and $\mathbb{F}_{26}$.
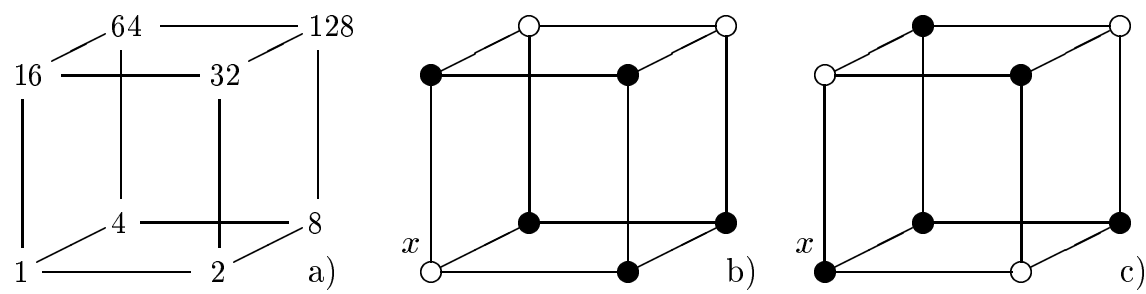

Figure 5: (a) Weights used for the coding of the pixel configurations of a 3d image, (b) and (c) pixel configurations coded as $g(x)=62$ and $g(x)=109$, respectively. In these examples the corresponding component $w_{i}$ of the vector $w$ in (11) depends considerably on the chosen adjacency. We get the values $-3,-3,-7$, and 1 for the configuration (b) and $-1,-5,-1$, and 3 for (c), respectively, see also Table 1 .

\subsection{Pairs of complementary adjacencies}

It is a usual requirement in image analysis that any adjacency for the 'object' $X$ has to be in coherence and consistency with an appropriate 'complementary adjacency' used for the 'background' $X^{c}$. Usually, the criterion is some discrete Jordan surface theorem. Our motivation is consistency (6) of the approximations of the Euler number.

Definition 5. The pair $\left(\mathbb{F}(\mathcal{G}), \mathbb{F}\left(\mathcal{G}_{c}\right)\right)$ of adjacency systems is complementary if for all bounded sets $X \subset \mathbb{R}^{n}$ 
(i) $F \cap F_{c}=\emptyset$ for all $F \in \mathbb{F}(\mathcal{G}) \sqcap X$ and $F_{c} \in \mathbb{F}\left(\mathcal{G}_{c}\right) \sqcap X^{c}$ and

(ii) $\tilde{\chi}^{n}(\mathbb{F}(\mathcal{G}) \sqcap X)=(-1)^{n+1} \tilde{\chi}^{n}\left(\mathbb{F}\left(\mathcal{G}_{c}\right) \sqcap X^{c}\right)$.

Notice that (i) is a nontrivial requirement since it is not even allowed that the discretizations of $X$ and of $X^{c}$ - as topologically closed polyhedra - touch in any point. Also (ii) is not an obvious consequence of (1) since the discretizations of $X$ and $X^{c}$ do not fill the space.

Rremark 4. Herman introduced in [5] the concept of Jordan pair. Two adjacencies are a Jordan pair if endowing object and background with these adjacencies yields a digital Jordan surface theorem. (Very roughly, a closed surface divides the space properly into two components. Paradoxes as in [5, Proposition 3.1] are prevented.) In [5, 10, 12, 26] Jordan surface theorems are proved for different concepts of digital surface and the pairs $(6,26),(26,6)$, $(6,18)$, and $(18,6)$, in $[12]$ also for $(14,6),(6,14)$. The surface concept in $[5,12,26]$ is motivated by surface rendering algorithms and not suitable in our context. However, provided the right definition of a surface, we assume that a Jordan surface theorem holds for our $(14.1,14.1)$ and $(14.2,14.2)$, too. However, this is beyond the scope of this paper and will be investigated in a future paper.

An important consequence of $(i)$ is that an edge of $\mathbb{F}$ and an edge of $\mathbb{F}_{c}$ may either coincide or meet in a lattice point or be disjoint, but never may intersect in a single point not being a lattice point. This limits the choice of diagonals on the facets of the lattice cells as well as of spatial diagonals through the cells. (In the $2 \mathrm{~d}$ case the 8 neighborhood can only be accompanied by the 4 neighborhood, since in the 8 neighborhood already both diagonals are edges in the corresponding $\mathbb{F}$.)

On the other hand, (ii) demands some kind of maximization of the sets of edges and of the refinement of the tessellation of one of the adjacencies if the complementary one is given. E.g. it has to be guaranteed that two points of the discretized set $\mathbb{F} \sqcap X$ are either connected within this set or they are separated by the discretization $\mathbb{F}_{c} \sqcap X^{c}$ of the complement. (In the $2 \mathrm{~d}$ case the 4 neighborhood has to be combined with the 8 neighborhood for the complement in order to fulfill (ii).)

It is not yet clear whether there exists a complementary adjacency for every adjacency and whether this would be unique. However, it is easy to see that if $\mathbb{F}\left(\mathcal{G}_{c}\right)$ is a complementary adjacency of $\mathbb{F}(\mathcal{G})$ then also $\mathbb{F}(\mathcal{G})$ is complementary to $\mathbb{F}\left(\mathcal{G}_{c}\right)$. An adjacency system $\mathbb{F}(\mathcal{G})$ is said to be self-complementary if $\tilde{\chi}^{n}\left(\mathbb{F}(\mathcal{G}) \sqcap X^{c}\right)=(-1)^{n+1} \tilde{\chi}^{n}(\mathbb{F}(\mathcal{G}) \sqcap X)$ for all $X$.

Theorem 3. The pair $\left(\mathbb{F}_{6}, \mathbb{F}_{26}\right)$ of adjacency systems is a pair complementary adjacency systems, and $\left(\mathbb{F}_{2} 6, \mathbb{F}_{6}\right)$ is complementary, too.

Proof: In order to show (i) of Definition 5 note that $\left(X \cap \mathbb{L}^{3}\right) \cap\left(X^{c} \cap \mathbb{L}^{3}\right)=\emptyset$, i.e. the sets of vertices of the discretizations of $X$ and of $X^{c}$ are disjoint. Since the edges of $\mathbb{F}_{6}$ either coincide or meet in an edge or are disjoint to all edges of $\mathbb{F}_{26}$ we can conclude that the union of all edges of the discretization of $X$ is disjoint to that of $X^{c}$. From the fact that the tessellations of $\mathbb{F}_{26}$ are refinements of the tessellation $\mathcal{G}_{0}$ one can now derive (i). Namely, since each $2 \mathrm{~d}$ face belonging to $\mathbb{F}_{6}$ is the union of $2 \mathrm{~d}$ faces in $\mathbb{F}_{26}$, it is clear that if a $2 \mathrm{~d}$ face belongs to $\mathbb{F}_{6} \sqcap X^{c}$ then no subset of it (of any dimension) may belong to $\mathbb{F}_{26} \sqcap X^{c}$. Analogous arguments can be used for the $3 \mathrm{~d}$ cells.

Due to the additivity of the Euler number it is sufficient to show that the consistency condition (ii) in Definition 5 is fulfilled in each cell of the lattice. This can be checked considering all 256 possible point configurations on the vertices of a cell. Of course, it has to 
be taken into account that each $2 \mathrm{~d}$ facet of a cell belongs to two cells, each edge belongs to four cells and each vertex belongs to 8 cells. We use the same edge correction as in (10).

It is sufficient for the validity of the consistency relation (ii) that $w_{i}^{(a)}=w_{255-i}^{(d)}$ for $i=1, \ldots, 128$, see Tables 1 and 2 .

\begin{tabular}{r|rrrr}
$i$ & $(\mathrm{a})$ & $(\mathrm{b})$ & $(\mathrm{c})$ & $(\mathrm{d})$ \\
\hline 0 & 0 & 0 & 0 & 0 \\
1 & 1 & 1 & 1 & 1 \\
2 & 1 & 1 & 1 & 1 \\
3 & 0 & 0 & 0 & 0 \\
4 & 1 & 1 & 1 & 1 \\
5 & 0 & 0 & 0 & 0 \\
6 & 2 & 2 & 2 & -2 \\
7 & -1 & -1 & -1 & -1 \\
8 & 1 & 1 & 1 & 1 \\
9 & 2 & -2 & -2 & -2 \\
10 & 0 & 0 & 0 & 0 \\
11 & -1 & -1 & -1 & -1 \\
12 & 0 & 0 & 0 & 0 \\
13 & -1 & -1 & -1 & -1 \\
14 & -1 & -1 & -1 & -1 \\
15 & 0 & 0 & 0 & 0 \\
16 & 1 & 1 & 1 & 1 \\
17 & 0 & 0 & 0 & 0 \\
18 & 2 & 2 & 2 & -2 \\
19 & -1 & -1 & -1 & -1 \\
20 & 2 & 2 & -2 & -2 \\
21 & -1 & -1 & -1 & -1 \\
22 & 3 & 3 & -1 & -1 \\
23 & -2 & -2 & -2 & -2 \\
24 & 2 & 2 & 2 & -6 \\
25 & 1 & -3 & -3 & -3 \\
26 & 1 & 1 & 1 & -3 \\
27 & -2 & -2 & -2 & -2 \\
28 & 1 & 1 & -3 & -3 \\
29 & -2 & -2 & -2 & -2 \\
30 & 0 & 0 & -4 & 0 \\
31 & -1 & -1 & -1 & -1 \\
& & &
\end{tabular}

\begin{tabular}{r|rrrr}
$i$ & $(\mathrm{a})$ & $(\mathrm{b})$ & $(\mathrm{c})$ & $(\mathrm{d})$ \\
\hline 32 & 1 & 1 & 1 & 1 \\
33 & 2 & -2 & -2 & -2 \\
34 & 0 & 0 & 0 & 0 \\
35 & -1 & -1 & -1 & -1 \\
36 & 2 & 2 & 2 & -6 \\
37 & 1 & -3 & -3 & -3 \\
38 & 1 & 1 & 1 & -3 \\
39 & -2 & -2 & -2 & -2 \\
40 & 2 & 2 & -2 & -2 \\
41 & 3 & -5 & -1 & -1 \\
42 & -1 & -1 & -1 & -1 \\
43 & -2 & -2 & -2 & -2 \\
44 & 1 & 1 & -3 & -3 \\
45 & 0 & -4 & 0 & 0 \\
46 & -2 & -2 & -2 & -2 \\
47 & -1 & -1 & -1 & -1 \\
48 & 0 & 0 & 0 & 0 \\
49 & -1 & -1 & -1 & -1 \\
50 & -1 & -1 & -1 & -1 \\
51 & 0 & 0 & 0 & 0 \\
52 & 1 & 1 & -3 & -3 \\
53 & -2 & -2 & -2 & -2 \\
54 & 0 & 0 & -4 & 0 \\
55 & -1 & -1 & -1 & -1 \\
56 & 1 & 1 & -3 & -3 \\
57 & 0 & -4 & 0 & 0 \\
58 & -2 & -2 & -2 & -2 \\
59 & -1 & -1 & -1 & -1 \\
60 & 0 & 0 & -8 & 0 \\
61 & -3 & -3 & 1 & 1 \\
62 & -3 & -3 & -7 & 1 \\
63 & 0 & 0 & 0 & 0
\end{tabular}

\begin{tabular}{r|rrrr}
$i$ & $(\mathrm{a})$ & $(\mathrm{b})$ & $(\mathrm{c})$ & $(\mathrm{d})$ \\
\hline 64 & 1 & 1 & 1 & 1 \\
65 & 2 & -2 & 2 & -2 \\
66 & 2 & 2 & 2 & -6 \\
67 & 1 & -3 & 1 & -3 \\
68 & 0 & 0 & 0 & 0 \\
69 & -1 & -1 & -1 & -1 \\
70 & 1 & 1 & 1 & -3 \\
71 & -2 & -2 & -2 & -2 \\
72 & 2 & 2 & 2 & -2 \\
73 & 3 & -5 & -1 & -1 \\
74 & 1 & 1 & 1 & -3 \\
75 & 0 & -4 & 0 & 0 \\
76 & -1 & -1 & -1 & -1 \\
77 & -2 & -2 & -2 & -2 \\
78 & -2 & -2 & -2 & -2 \\
79 & -1 & -1 & -1 & -1 \\
80 & 0 & 0 & 0 & 0 \\
81 & -1 & -1 & -1 & -1 \\
82 & 1 & 1 & 1 & -3 \\
83 & -2 & -2 & -2 & -2 \\
84 & -1 & -1 & -1 & -1 \\
85 & 0 & 0 & 0 & 0 \\
86 & 0 & 0 & 0 & 0 \\
87 & -1 & -1 & -1 & -1 \\
88 & 1 & 1 & 1 & -3 \\
89 & 0 & -4 & -4 & 0 \\
90 & 0 & 0 & 0 & 0 \\
91 & -3 & -3 & -3 & 1 \\
92 & -2 & -2 & -2 & -2 \\
93 & -1 & -1 & -1 & -1 \\
94 & -3 & -3 & -3 & 1 \\
95 & 0 & 0 & 0 & 0
\end{tabular}

\begin{tabular}{r|rrrr}
$i$ & $(\mathrm{a})$ & $(\mathrm{b})$ & $(\mathrm{c})$ & $(\mathrm{d})$ \\
\hline 96 & 2 & 2 & 2 & -2 \\
97 & 3 & -5 & -1 & -1 \\
98 & 1 & 1 & 1 & -3 \\
99 & 0 & -4 & 0 & 0 \\
100 & 1 & 1 & 1 & -3 \\
101 & 0 & -4 & -4 & 0 \\
102 & 0 & 0 & 0 & 0 \\
103 & -3 & -3 & -3 & 1 \\
104 & 3 & 3 & -1 & -1 \\
105 & 4 & -8 & 0 & 4 \\
106 & 0 & 0 & 0 & 0 \\
107 & -1 & -5 & -1 & 3 \\
108 & 0 & 0 & -4 & 0 \\
109 & -1 & -5 & -1 & 3 \\
110 & -3 & -3 & -3 & 1 \\
111 & -2 & -2 & -2 & 2 \\
112 & -1 & -1 & -1 & -1 \\
113 & -2 & -2 & -2 & -2 \\
114 & -2 & -2 & -2 & -2 \\
115 & -1 & -1 & -1 & -1 \\
116 & -2 & -2 & -2 & -2 \\
117 & -1 & -1 & -1 & -1 \\
118 & -3 & -3 & -3 & 1 \\
119 & 0 & 0 & 0 & 0 \\
120 & 0 & 0 & -4 & 0 \\
121 & -1 & -5 & -1 & 3 \\
122 & -3 & -3 & -3 & 1 \\
123 & -2 & -2 & -2 & 2 \\
124 & -3 & -3 & -7 & 1 \\
125 & -2 & -2 & 2 & 2 \\
126 & -6 & -6 & -6 & 2 \\
127 & 1 & 1 & 1 & 1
\end{tabular}

Table 1: The components $w_{i}, i=0, \ldots, 127$, of the vector $w$ : column (a) for the adjacency $\mathbb{F}_{6}$, (b) for $\mathbb{F}_{14.1},(\mathrm{c})$ for $\mathbb{F}_{14.2}$, and (d) for $\mathbb{F}_{26}$.

Theorem 4. The pairs $\left(\mathbb{F}_{14.1}, \mathbb{F}_{14.1}\right)$ and $\left(\mathbb{F}_{14.2}, \mathbb{F}_{14.2}\right)$ are complementary pairs of adjacency systems.

Proof: Condition (i) of Definition 5 can be proved using the same arguments as for the $(6,26)$ neighborhood. To prove the consistency (ii), we consider the possible point configurations in single cells as in the proof for the $(6,26)$ adjacencies. The evaluations in columns (b) and (c) of Tables 1 and 2 show that $w_{i}^{(b)}=w_{255-i}^{(b)}$ for $\mathbb{F}_{14.1}$ and $w_{i}^{(c)}=w_{255-i}^{(c)}$ for $\mathbb{F}_{14.2}$.

Intuitively, the reason for the self-complementarity of $\mathbb{F}_{14.1}$ as well as of $\mathbb{F}_{14.2}$ is the fact that a tetrahedral tessellation cannot be further refined and that also the induced tessellations 
on the $2 \mathrm{~d}$ facets of the cells are translation invariant, i.e. the tessellations on the contact faces of adjacent cells coincide. This is not true for all tetrahedral tessellations. A counterexample is, for instance, any tessellation containing the tetrahedron with the vertices $x_{0}, x_{3}, x_{5}, x_{6}$.

\begin{tabular}{|c|c|c|c|c|c|c|c|c|c|c|c|c|c|c|c|c|c|c|c|}
\hline$i$ & (a) & (b) & (c) & (d) & $i$ & (a) & (b) & (c) & (d) & $i$ & (a) & (b) & (c) & (d) & $i$ & \multicolumn{4}{|c|}{$(\mathrm{a})(\mathrm{b})(\mathrm{c})(\mathrm{d})$} \\
\hline 128 & 1 & 1 & 1 & 1 & 160 & 0 & 0 & 0 & 0 & 192 & 0 & 0 & 0 & 0 & 224 & -1 & -1 & -1 & -1 \\
\hline 129 & 2 & -6 & -6 & -6 & 161 & 1 & -3 & -3 & -3 & 193 & 1 & -3 & -7 & -3 & 225 & 0 & 0 & -4 & 0 \\
\hline 130 & 2 & -2 & 2 & -2 & 162 & -1 & -1 & -1 & -1 & 194 & 1 & -3 & 1 & -3 & 226 & -2 & -2 & -2 & -2 \\
\hline 131 & 1 & -3 & -7 & -3 & 163 & -2 & -2 & -2 & -2 & 195 & 0 & 0 & -8 & 0 & 227 & -3 & 1 & -3 & 1 \\
\hline 132 & 2 & -2 & -2 & -2 & 164 & 1 & -3 & -3 & -3 & 196 & -1 & -1 & -1 & -1 & 228 & -2 & -2 & -2 & -2 \\
\hline 133 & 1 & -3 & -3 & -3 & 165 & 0 & 0 & 0 & 0 & 197 & -2 & -2 & -2 & -2 & 229 & -3 & 1 & 1 & 1 \\
\hline 134 & 3 & -5 & -1 & -1 & 166 & 0 & -4 & -4 & 0 & 198 & 0 & -4 & 0 & 0 & 230 & -3 & -3 & -3 & 1 \\
\hline 135 & 0 & 0 & -4 & 0 & 167 & -3 & 1 & 1 & 1 & 199 & -3 & 1 & -3 & 1 & 231 & -6 & 2 & 2 & 2 \\
\hline 136 & 0 & 0 & 0 & 0 & 168 & -1 & -1 & -1 & -1 & 200 & -1 & -1 & -1 & -1 & 232 & -2 & -2 & -2 & -2 \\
\hline 137 & 1 & -3 & -3 & -3 & 169 & 0 & 0 & 0 & 0 & 201 & 0 & 0 & -4 & 0 & 233 & -1 & 3 & -1 & 3 \\
\hline 138 & -1 & -1 & -1 & -1 & 170 & 0 & 0 & 0 & 0 & 202 & -2 & -2 & -2 & -2 & 234 & -1 & -1 & -1 & -1 \\
\hline 139 & -2 & -2 & -2 & -2 & 171 & -1 & -1 & -1 & -1 & 203 & -3 & 1 & -3 & 1 & 235 & -2 & 2 & -2 & 2 \\
\hline 140 & -1 & -1 & -1 & -1 & 172 & -2 & -2 & -2 & -2 & 204 & 0 & 0 & 0 & 0 & 236 & -1 & -1 & -1 & -1 \\
\hline 141 & -2 & -2 & -2 & -2 & 173 & -3 & 1 & 1 & 1 & 205 & -1 & -1 & -1 & -1 & 237 & -2 & 2 & 2 & 2 \\
\hline 142 & -2 & -2 & -2 & -2 & 174 & -1 & -1 & -1 & -1 & 206 & -1 & -1 & -1 & -1 & 238 & 0 & 0 & 0 & 0 \\
\hline 143 & -1 & -1 & -1 & -1 & 175 & 0 & 0 & 0 & 0 & 207 & 0 & 0 & 0 & 0 & 239 & 1 & 1 & 1 & 1 \\
\hline 144 & 2 & -2 & -2 & -2 & 176 & -1 & -1 & -1 & -1 & 208 & -1 & -1 & -1 & -1 & 240 & 0 & 0 & 0 & 0 \\
\hline 145 & 1 & -3 & -3 & -3 & 177 & -2 & -2 & -2 & -2 & 209 & -2 & -2 & -2 & -2 & 241 & -1 & -1 & -1 & -1 \\
\hline 146 & 3 & -5 & -1 & -1 & 178 & -2 & -2 & -2 & -2 & 210 & 0 & -4 & 0 & 0 & 242 & -1 & -1 & -1 & -1 \\
\hline 147 & 0 & 0 & -4 & 0 & 179 & -1 & -1 & -1 & -1 & 211 & -3 & 1 & -3 & 1 & 243 & 0 & 0 & 0 & 0 \\
\hline 148 & 3 & -5 & -1 & -1 & 180 & 0 & -4 & 0 & 0 & 212 & -2 & -2 & -2 & -2 & 244 & -1 & -1 & -1 & -1 \\
\hline 149 & 0 & 0 & 0 & 0 & 181 & -3 & 1 & 1 & 1 & 213 & -1 & -1 & -1 & -1 & 245 & 0 & 0 & 0 & 0 \\
\hline 150 & 4 & -8 & 0 & 4 & 182 & -1 & -5 & -1 & 3 & 214 & -1 & -5 & -1 & 3 & 246 & -2 & -2 & -2 & 2 \\
\hline 151 & -1 & 3 & -1 & 3 & 183 & -2 & 2 & 2 & 2 & 215 & -2 & 2 & -2 & 2 & 247 & 1 & 1 & 1 & 1 \\
\hline 152 & 1 & -3 & -3 & -3 & 184 & -2 & -2 & -2 & -2 & 216 & -2 & -2 & -2 & -2 & 248 & -1 & -1 & -1 & -1 \\
\hline 153 & 0 & 0 & 0 & 0 & 185 & -3 & 1 & 1 & 1 & 217 & -3 & 1 & 1 & 1 & 249 & -2 & 2 & 2 & 2 \\
\hline 154 & 0 & -4 & -4 & 0 & 186 & -1 & -1 & -1 & -1 & 218 & -3 & -3 & -3 & 1 & 250 & 0 & 0 & 0 & 0 \\
\hline 155 & -3 & 1 & 1 & 1 & 187 & 0 & 0 & 0 & 0 & 219 & -6 & 2 & 2 & 2 & 251 & 1 & 1 & 1 & 1 \\
\hline 156 & 0 & -4 & 0 & 0 & 188 & -3 & -3 & 1 & 1 & 220 & -1 & -1 & -1 & -1 & 252 & 0 & 0 & 0 & 0 \\
\hline 157 & -3 & 1 & 1 & 1 & 189 & -6 & 2 & 2 & 2 & 221 & 0 & 0 & 0 & 0 & 253 & 1 & 1 & 1 & 1 \\
\hline 158 & -1 & -5 & -1 & 3 & 190 & -2 & -2 & 2 & 2 & 222 & -2 & -2 & -2 & 2 & 254 & 1 & 1 & 1 & 1 \\
\hline 159 & -2 & 2 & 2 & 2 & 191 & 1 & 1 & 1 & 1 & 223 & 1 & 1 & 1 & 1 & 255 & 0 & 0 & 0 & 0 \\
\hline
\end{tabular}

Table 2: The components $w_{i}, i=128, \ldots, 255$, of the vector $w$ : column (a) for the adjacency $\mathbb{F}_{6},(\mathrm{~b})$ for $\mathbb{F}_{14.1}$, (c) for $\mathbb{F}_{14.2}$, and (d) for $\mathbb{F}_{26}$.

\subsection{Sufficient conditions for correct approximation}

Consider a pair $\left(\mathbb{F}(\mathcal{G}), \mathbb{F}\left(\mathcal{G}_{c}\right)\right)$ of adjacencies with respect to the superpositions of admissible tessellations $\mathcal{G}$ and $\mathcal{G}_{c}$, respectively. Let $\mathcal{S}(\mathcal{G})$ denote the set of all segments $[x, y]$ for which the edge $\langle x, y\rangle$ belongs to the neighborhood graph $\Gamma$ of $\mathbb{F}(\mathcal{G})$.

Theorem 5. Let $\left(\mathbb{F}(\mathcal{G}), \mathbb{F}\left(\mathcal{G}_{c}\right)\right)$ be one of the four pairs of complementary adjacencies $\left(\mathbb{F}_{6}, \mathbb{F}_{26}\right),\left(\mathbb{F}_{26}, \mathbb{F}_{6}\right),\left(\mathbb{F}_{14.1}, \mathbb{F}_{14.1}\right)$ and $\left(\mathbb{F}_{14.2}, \mathbb{F}_{14.2}\right)$. If $X$ is morphologically closed with respect to all segments of $\mathcal{S}(\mathcal{G})$, and its complement $X^{c}$ is morphologically closed with respect to all segments of $\mathcal{S}\left(\mathcal{G}_{c}\right)$ then the approximation $\tilde{\chi}^{3}(\mathbb{F}(\mathcal{G}) \sqcap X)$ is correct for $\chi^{3}(X)$, i.e. 
$\tilde{\chi}^{3}(\mathbb{F}(\mathcal{G}) \sqcap X)=\chi^{3}(X)$

The proof will be given for $\mathbb{F}(\mathcal{G})=\mathbb{F}_{14.1}, \mathbb{F}_{14.2}$ and $\mathbb{F}_{26}$ respectively. The case $\mathbb{F}(\mathcal{G})=\mathbb{F}_{6}$ can be reduced to $\mathbb{F}(\mathcal{G})=\mathbb{F}_{26}$ for the complement with the help of the consistency relation (6). We write $\cup \mathbb{F}(\mathcal{G}) \sqcap X=\bigcup\{F \in \mathbb{F}(\mathcal{G}) \sqcap X\}$ for short. Due to the additivity of the Euler number, it is sufficient to prove $\tilde{\chi}^{k}(X \cap P)=\tilde{\chi}^{k}(\cup \mathbb{F}(\mathcal{G}) \sqcap X \cap P), k=0, \ldots 3$, where $P$ is an arbitrary tetrahedron of the tessellation according to the 14.1 or 14.2 adjacency, or a lattice cell $\bar{C}+x, x \in \mathbb{L}^{3}$ if $\mathbb{F}=\mathbb{F}_{26}$ is regarded, or a $k$-face of the mentioned polytope, respectively. In the following let $P$ be one of these particular polytopes of dimension $k$.

As a preliminary step we prove the following assertion.

Lemma 1. Under the assumptions of Theorem 5 we have

$$
X \cap P=\emptyset \Longleftrightarrow X \cap \mathcal{F}^{0}(P)=\emptyset
$$

and

$$
X \cap \mathcal{F}^{0}(P) \neq \emptyset \Longleftrightarrow \tilde{\chi}^{k}(\cup \mathbb{F}(\mathcal{G}) \sqcap X \cap P)=1 .
$$

Proof: The nontrivial part of (12) can be shown by induction over the dimension $j$ of the faces of $P$. If $X \cap \mathcal{F}^{0}(P)=\emptyset$, i.e. $\mathcal{F}^{0}(P) \subseteq X^{c}$, then the morphological closure with the edges of $P$ yields $\mathcal{F}^{1}(P) \subseteq X^{c}$. For those particular polytopes $P$ which are considered, this procedure of morphological closing can be iterated to show $P \subseteq X^{c}$.

The equivalence (13) is a consequence of $\cup \mathbb{F}(\mathcal{G}) \sqcap X \cap P=\operatorname{conv}\left(\mathcal{F}^{0}(P) \cap X\right)$, the convex hull of the vertices belonging to $X$. (Notice that this is true for $\mathbb{F}_{14.1}, \mathbb{F}_{14.2}, \mathbb{F}_{26}$ but not for $\mathbb{F}_{6}$.)

Proof of Theorem 5: Regarding the lemma above, it is sufficient to show that

$$
\tilde{\chi}^{k}(X \cap P)=1 \Longleftrightarrow X \cap P \neq \emptyset .
$$

This can be done with Hadwiger's recursive formula (3). The crucial point is that for any $j$-dimensional plane $L^{j}$ parallel to a $j$-dimensional face of $P$ and any $(j-1)$-dimensional plane $L \subset L^{j}$ ( $L$ can be regarded as a linear subspace in $L^{j}$ ) parallel to a $(j-1)$-dimensional face of $P$

$$
\begin{gathered}
\tilde{\chi}^{j}\left(X \cap P \cap L^{j}\right)= \\
\sum_{x \in L^{\perp} \cap L^{j}} \lim _{\varepsilon \rightarrow 0}\left[\tilde{\chi}^{j-1}\left(X \cap P \cap L_{x}\right)-\tilde{\chi}^{j-1}\left(X \cap P \cap L_{x+\varepsilon u}\right)\right]=1
\end{gathered}
$$

if $X \cap P \cap L^{j} \neq \emptyset$, with $u \in L^{\perp} \cap L^{j}$ and $j=1, \ldots, k$.

This formula will now be shown in the following parts (a) to (d).

(a) The assumption that $X^{c}$ is morphologically closed with respect to all segments in $\mathcal{S}\left(\mathcal{G}_{c}\right)$ implies:

For all $y \in X$ and all $F \in \mathcal{S}\left(\mathcal{G}_{c}\right)$ there exists a $t \in \mathbb{R}^{3}$ with

$$
y \in(F+t) \subseteq X,
$$

and analogously, if $X$ is morphologically closed with respect to all segments in $\mathcal{S}(\mathcal{G})$, then

$$
\text { for all } y \in X^{c} \text { and all } F \in \mathcal{S}(\mathcal{G}) \text { there exists a } t \in \mathbb{R}^{3} \text { with }
$$

$$
y \in(F+t) \subseteq X^{c},
$$


see [30], II.D., relations between morphologically closed and open sets and the characterization of morphologically open sets.

(b) Let $L^{j}$ and $L$ be the same as used in (15) and $x_{1}, x_{2}, x \in L^{\perp} \cap L^{j}$. If $P \cap L_{x_{1}} \neq \emptyset$ and $P \cap L_{x_{2}} \neq \emptyset$ then

$$
\begin{gathered}
X \cap P \cap L_{x_{1}}=\emptyset \wedge X \cap P \cap L_{x_{2}}=\emptyset \Longrightarrow \\
X \cap P \cap L_{x}=\emptyset \text { for all } L_{x} \text { between } L_{x_{1}}, L_{x_{2}} .
\end{gathered}
$$

Assume $X \cap P \cap L_{x} \neq \emptyset$, i.e. there is a $y \in X \cap P \cap L_{x}$. Then (16) provides that there is a segment $y \in(F+t) \subseteq X$. This segment can be chosen as a translate of an edge of $P$ which hits $L_{x_{1}}$ and $L_{x_{2}}$. Hence $(F+t)$ hits at least one of the sets $L_{x_{1}} \cap P$ or $L_{x_{2}} \cap P$. This contradicts the assumption, and thus (18) is shown.

(c) With the same notation as in (b) and analogously to (18)

$$
\begin{gathered}
X \cap P \cap L_{x_{1}} \neq \emptyset \wedge X \cap P \cap L_{x_{2}} \neq \emptyset \Longrightarrow \\
X \cap P \cap L_{x} \neq \emptyset \text { for all } L_{x} \text { between } L_{x_{1}}, L_{x_{2}} .
\end{gathered}
$$

Let $y_{1} \in X \cap P \cap L_{x_{1}}, y_{2} \in X \cap P \cap L_{x_{2}}$.

Firstly consider the case that $L_{x_{1}}, L_{x_{2}} \subset L^{2}$ are lines. Let $S \subseteq P$ be the smallest triangle in the plane $L^{2}$ with $y_{2}$ as one vertex and $y_{1}$ an element in the opposite edge, say $F_{S}$ which is parallel to an edge $F \in \mathcal{S}\left(\mathcal{G}_{c}\right)$, and all edges of $S$ parallel to one of the segments in $\mathcal{S}(\mathcal{G})$. (Notice that it is not a misprint that once the index $c$ occurs and once not. This distinction is important for the pair of adjacencies $(26,6)$.) Then, according to (16) there is a translated segment $F+t \subseteq X$ through $y_{1}$. Since $F_{S}$ is not longer than $F$, the translate $F+t$ hits at least one of the endpoints of $F_{S}$, say $y_{S}$. Since $X$ is assumed to be morphologically closed with respect to all segments in $\mathcal{S}(\mathcal{G})$, also the segment $\left[y_{2}, y_{S}\right] \subseteq X$ since this is not longer than the parallel segment from $\mathcal{S}(\mathcal{G})$. Thus it is shown that the points $y_{1}$ and $y_{2}$ are connected by a chain of segments $\left[y_{1}, y_{S}\right]$ and $\left[y_{S}, y_{2}\right]$ which belong to $X \cap P$. This yields (19).

If $L_{x_{1}}, L_{x_{2}}$ are planes, then a minimal tetrahedron $S$ exists with $y_{2}$ as one vertex and $y_{1}$ in the opposite facet of $S$, and all edges parallel to appropriate segments in $\mathcal{S}(\mathcal{G})$. With an iterated application of the same ideas and arguments as they were used for the triangle above, it is possible to show the existence of a sequence of segments inside $X \cap P$ which connects $y_{1}$ and $y_{2}$.

(d) Summarizing (b) and (c) implies that $\left\{x \in L^{\perp} \cap L^{j}: X \cap P \cap L_{x} \neq \emptyset\right\}$ is a closed convex set, i.e. a segment on the line $L^{\perp} \cap L^{j}$. Hence, if $X \cap P \cap L^{j} \neq \emptyset$, the sum in (15) has exactly one item different from 0 , and its value is 1 . Induction over $j=1, \ldots, k$ yields (15) and the proof of (14) is complete.

If nothing can be assumed about the regularity of $X$ or $X^{c}$, both $X \cap \mathbb{L}^{n}$ and $X^{c} \cap \mathbb{L}^{n}$ should be treated equally. In particular, self-complementary adjacencies can be a good choice. On the other hand, our simulation studies in Section 4 illustrate that the self-complementary 14.1 and 14.2 adjacencies can induce a considerably large approximation error.

Assume now for example that $X$ is a finite union of compact and convex sets $X_{i}, i=$ $1, \ldots, m$, having smooth surfaces. If the $X_{i}$ are morphologically open with respect to the segments of $\mathcal{S}\left(\mathcal{G}_{c}\right)$ then their union $X=\cup_{i=1}^{m} X_{i}$ is morphologically open with respect to these segments, too. But $X^{c}$ is not necessarily open with respect to the elements of $\mathcal{S}\left(\mathcal{G}_{c}\right)$. As a 
consequence, the pair $\left(\mathbb{F}_{6}, \mathbb{F}_{26}\right)$ of adjacency systems is the best choice for the measurement of $\chi^{n}(X)$; i.e. $\tilde{\chi}^{n}\left(\mathbb{F}_{6} \sqcap X\right)$ is 'better' than the approximation of $\chi^{n}(X)$ with respect to a self-complementary adjacency system.

The following lemma states that, if both $X$ and $X^{c}$ are 'regular enough', their Euler number is independent of the choice of the adjacency system.

Lemma 2. Let $X$ be a compact set which is morphologically open and morphologically closed with respect to all segments $[x, y]$ with $x, y \in \mathcal{F}^{0}(\bar{C}), x \neq y$, and $(x, y) \subset C$. Then $\tilde{\chi}^{n}(\mathbb{F}(\mathcal{G}) \sqcap X)=\chi^{n}(X)$ for all superpositions of admissible tessellations $\mathcal{G}$.

Proof: Notice that the set of segments mentioned in this lemma is equivalent to $\mathcal{S}\left(\mathcal{G}_{\max }\right)$. The proof follows from Theorem 5 , since $\mathcal{S}(\mathcal{G}) \subset \mathcal{S}\left(\mathcal{G}_{\max }\right)$.

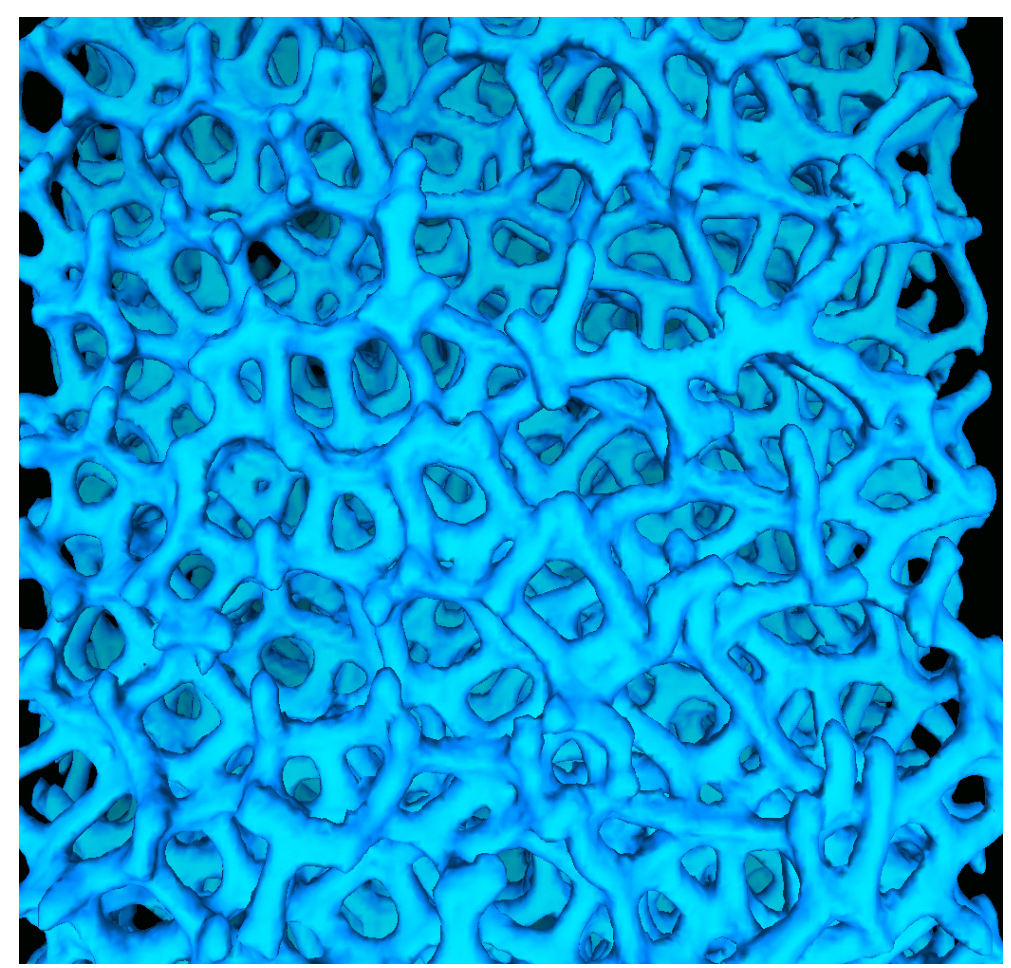

Figure 6: The microstructure of an open nickel foam. The XCT scan consists of $341 \times 320 \times 511$ pixels (simple cubic lattice, $|u|=10 \mu \mathrm{m}$ ).

\section{Applications and a simulation study}

As applications we consider the microstructures of three porous materials: a Fontainebleau sandstone, an open nickel foam, and an autoclaved aerated concrete (AAC). Samples of these microstructures are given as 3d images. For visualizations of the data see Figures 1, 6, and 7 , respectively. The Fontainebleau sandstone is a standard example in papers on simulation of fluid flow through porous media. (Notice that different data sets are used.) The three microstructures are given as $3 \mathrm{~d}$ images obtained by X-ray microtomography. 


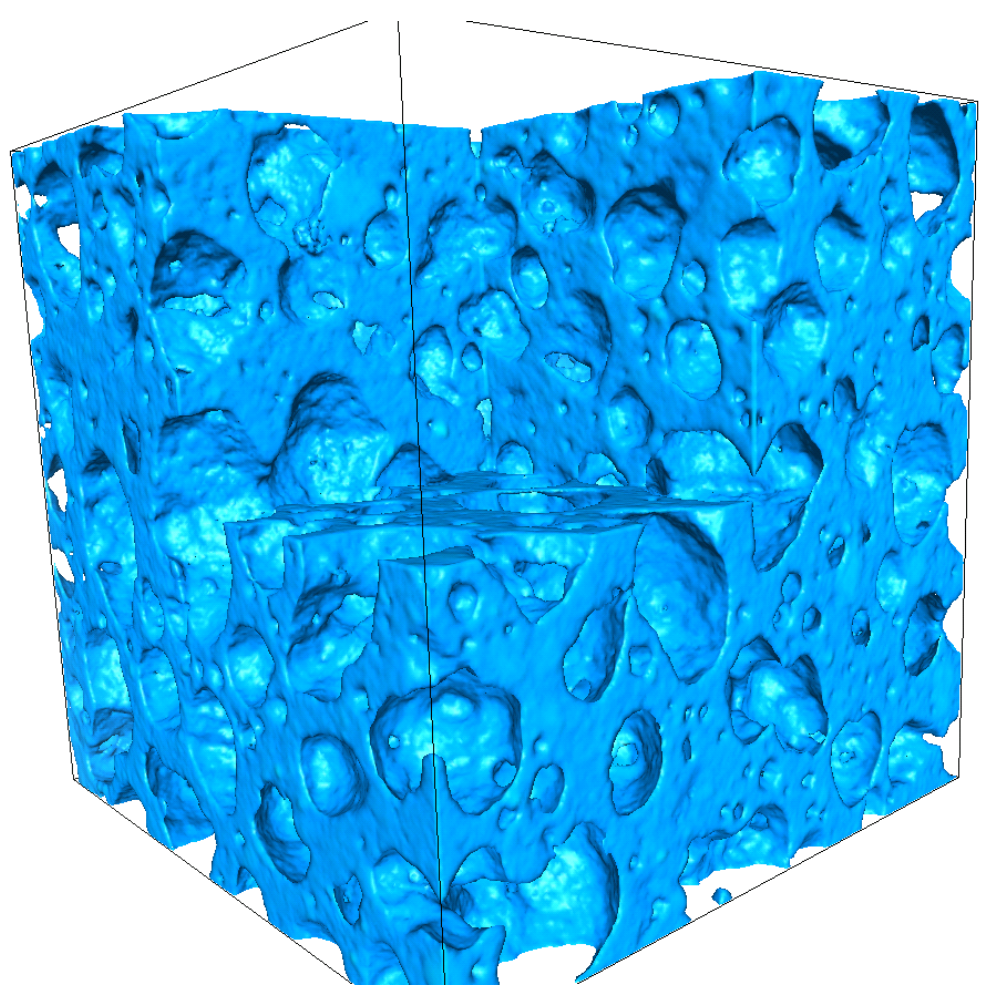

Figure 7: The microstructure of an autoclaved aerated concrete (AAC). The XCT scan consists of $320 \times 330 \times 330$ pixels (simple cubic lattice, $|u|=31.3 \mu \mathrm{m}$ ).

Each component of the considered microstructures can be modeled as a macroscopically homogeneous random set $\Xi$ as described in [23]. More precisely, we assume that with probability one the random set $\Xi \cap W$ belongs to the convex ring (for every compact set $W$ with nonempty interior), and the distribution law of $\Xi$ is invariant with respect to translations $x \in \mathbb{R}^{n}$. Then the density of the Euler number (which is also called the connectivity density) is given by

$$
\chi_{V}(\Xi)=\lim _{\varepsilon \rightarrow \infty} \frac{\tilde{\chi}^{n}(\mathbb{F} \sqcap(\Xi \cap \varepsilon W))}{|\varepsilon W|} .
$$

Here $|\cdot|$ denotes $n$-dimensional volume. The density of the Euler number can be estimated from the set $\Xi$ observed in a compact window $W$ using

$$
\widehat{\chi V}(\Xi)=\frac{1}{\#\left(\mathbb{L}^{n} \cap W_{0}\right)|C|} \sum_{x \in \mathbb{L}^{n} \cap W_{0}} \sum_{k=0}^{n}(-1)^{k} \sum_{\ell=k}^{n} 2^{\ell-n} \#\left(\mathcal{F}^{k \ell} \sqcap \Xi_{-x}\right),
$$

where $W_{0}$ is the reduced window, $W_{0}=W \ominus \check{C}$. Notice that $\#\left(\mathbb{L}^{n} \cap W_{0}\right)|C| \approx\left|W_{0}\right|$. Furthermore, we remark that the estimator $\widehat{\chi_{V}}(\Xi)$ may be biased but it is free of edge effects.

Table 3 shows that experimental values for $\chi_{V}(\Xi)$ can depend highly on the chosen adjacency. Differences in the measurement values are a consequence of the irregularity of the microstructures. It is an obvious consequence of Theorem 5 and Lemma 2 that the constituents of the microstructures are not morphologically regular with respect to the edges, face diagonals, and space diagonals of $C$. 


\begin{tabular}{l|cccc|c} 
microstructure/ geometric model & $\mathbb{F}_{6}$ & $\mathbb{F}_{14.1}$ & $\mathbb{F}_{14.2}$ & $\mathbb{F}_{26}$ & true value \\
\hline Fontainebleau sandstone, Figure 1 & -198 & -273 & -366 & -369 & unknown \\
open nickel foam, Figure 6 & -105 & -105 & -106 & -113 & unknown \\
AAC, Figure 7 & 4.65 & 5.46 & 5.09 & 6.21 & unknown \\
\hline Boolean model, $\lambda=500 \mathrm{~mm}^{-3}$ & 50 & -46 & -108 & -76 & 107 \\
Boolean model, $\lambda=1000 \mathrm{~mm}^{-3}$ & -308 & -515 & -679 & -592 & -188 \\
Boolean model, $\lambda=2000 \mathrm{~mm}^{-3}$ & -861 & -1079 & -1420 & -979 & -970 \\
Boolean model, $\lambda=4000 \mathrm{~mm}^{-3}$ & -365 & -261 & -579 & 240 & -603
\end{tabular}

Table 3: Experimental data for the density $\chi_{V}$ of the Euler number with respect to the adjacencies $\mathbb{F}_{6}, \mathbb{F}_{14.1}, \mathbb{F}_{14.2}$, and $\mathbb{F}_{26}$ for some microstructures and realizations of Boolean models. The data are given in $\mathrm{mm}^{-3}$. For the microstructures the true values are unknown. For the Boolean models the true values (expectations) were determined from the model parameters using Miles' formulae.

In the first example we consider the Fontainebleau sandstone shown in Figure 1. The 3d data are equivalent to that considered in [6]. In particular, for these data our algorithm leads to large differences between the estimates and, hence, they are only of low value for practical use. Obviously, the lateral resolution in the image is not sufficient for an accurate analysis of this very 'tiny' microstructure, i.e. the regularity conditions formulated in Theorem 5 are not fulfilled. In this case the image acquisition (i.e. the discretization) induced a certain loss of information with considerable consequences for the measurement of the Euler density.

For the open foam shown in Figure 6 the differences between the estimates are small. Since these differences are neglectable, one can believe that the foam is morphologically regular with respect to segments of lengths close to the lattice spacings. Thus we can also expect low bias of the estimates, i.e. the measurement values are assumed to be close to the true Euler density $\chi_{V}(\Xi)$.

Additionally to the analysis of these sample data we performed a simulation study. To compare theoretical (true) values of the density of the Euler number with estimates, we consider particular Boolean models in the Euclidean space for which the theoretical values can be computed from the model parameters using Miles' formulae, see [21],[30, p. 495], or [28, p. 217]. The Boolean models were simulated with balls of constant diameter $d=100 \mu \mathrm{m}$ and the densities $\lambda=500 \mathrm{~mm}^{-3}, 1000 \mathrm{~mm}^{-3}, 2000 \mathrm{~mm}^{-3}$, and $4000 \mathrm{~mm}^{-3}$ of the corresponding Poisson fields of germs. The corresponding realizations are observed on a simple cubic lattice with uniform spacings $|u|=10 \mu \mathrm{m}$. The experimental data were computed form 32 images (i.e. discretizations of simulated Boolean models) where each image was of size $512 \times 512 \times 512$ pixels (i.e. each sampling window was a cube of volume $134.2 \mathrm{~mm}^{3}$ ). The experimental values given in Table 3 are mean values taken over 32 images (realizations). The standard deviation of the means was less than $1.6 \mathrm{~mm}^{-3}$. This means that the distances of each experimental value from the corresponding true value can be considered as the bias of estimation. Notice that for large sampling windows, the estimation variance of the Euler-Poincaré characteristic of Boolean models can also be computed by formulae given in [4].

This simulation study shows that there can be a considerable bias in the estimates caused by discretization. It is perhaps surprising that a large bias can even occur in cases when the primary grains are much larger than the lattice cells. Notice that in our example the balls' diameter is $d=10|u|$. Thus the bias is mainly due to tiny structure in the complement $X^{c}$ when balls are very close together without overlapping.

Depending on the point density $\lambda$, the minimal bias is obtained for different adjacencies: $\mathbb{F}_{6}$ for $\lambda=500 \mathrm{~mm}^{-3}, 1000 \mathrm{~mm}^{-3}, \mathbb{F}_{26}$ for $\lambda=2000 \mathrm{~mm}^{-3}$, and $\mathbb{F}_{14.2}$ for $\lambda=4000 \mathrm{~mm}^{-3}$. 
That means, there is no clear advantage of one adjacency over the others and without further knowledge about the geometry of $\Xi$, an appropriate choice of $\mathbb{F}$ seems to be impossible.

In practical applications, the Euler number should be measured with respect to several adjacencies. Then the differences between the results provides an impression of the bias of the measurements. Of course, other adjacencies could be useful besides the four examples suggested in Subsection 3.3. Admissible tessellations and their superpositions yield a wide variety of adjacencies. A constructive method for finding complementary adjacencies has yet to be found.

\section{Acknowledgements}

The authors thank the editors for valuable hints on a first draft of this paper. We are indebted to Prof E. Schlegel for supplying the AAC specimen shown in Figure 7, S. Gondrom for scanning the nickel foam and the AAC, R. Hilfer for providing the data of the Fontainebleau sandstone, and H. Seibert for some ideas and helpful comments. The authors J.O and K.S. are grateful to the Stiftung Rheinland-Pfalz für Innovation for financial support.

\section{References}

[1] Bieri, H., W. Nef (1984): 'Alogorithms for the Euler Characteristic and Related Additive Functionals of Digital Objects'. Comput. Vision Graphics Image Process. 28, pp. 166175

[2] Gruber, P. M. (1993): 'Geometry of numbers'. In: Handbook of convex geometry, edited by P. M. Gruber, J. M. Wills (North Holland, Amsterdam), pp. 739-763

[3] Hadwiger, H. (1957): Vorlesungen über Inhalt, Oberfläche und Isoperimetrie (Springer, Heidelberg)

[4] Heinrich, L. (2002) 'Asymptodic normality of the Euler-Poincaré characteristic of Boolean models in large sampling windows.' Report No. 247, Universität Augsburg, Institut für Mathematik.

[5] Herman, G. T. (1992): 'Discrete Multidimensional Jordan Surfaces'. Graph. Mod. Image Proc. 54, pp. 507-515

[6] Hilfer, R. (2000): 'Local porosity theory and stochastic reconstruction'. In: Statistical Physics and Spatial Statistics, edited by K. R. Mecke, D. Stoyan (Springer, Heidelberg), pp. 203-241

[7] Imiya, A., U. Eckhardt (1997): 'The Euler Characteristic of Discrete Object'. Discrete Geometry for Computer Imagery , pp. 163-174

[8] Jernot, J. P., P. Jouannot-Chesney, C. Lantuéjoul (2001): 'Determination of the connectivity number of a set using a tessellation'. Manuscript.

[9] Khalimsky, E., R. Kopperman, P. Meyer (1990): 'Computer Graphics and connected topologies on finite ordered sets'. In: Topology and its Application, vol. 36 (Elsevier Science Publisher B.V), pp. 1-17

[10] Kong, T. Y., A. W. Roscoe (1985): 'Continuous analogs of axiomatized digital surfaces'. Comput. Vision Graphics Image Process. 29, pp. 60-86 
[11] Kong, T. Y., A. Rosenfeld (1989): 'Digital Topology: Introduction and Survey'. Comput. Vision Graphics Image Process. 48, pp. 357-393

[12] Kong, T. Y., J. K. Udupa (1992): 'A justification of a fast surface tracking algorithm'. CVGIP: Graph. Models Image Process. 54, pp. 162-170

[13] Lang, C., J. Ohser, R. Hilfer (2001): 'On the analysis of spatial binary images'. J. Microsc. 202, pp. 1-12

[14] Lee, C., T. Poston, A. Rosenfeld (1991): 'Winding and Euler Numbers for 2D and 3D Digital Images'. CVGIP: Graph. Models Image Process. 53(6), pp. 522-537

[15] Lee, C., T. Poston, A. Rosenfeld (1993): 'Holes and Genus of 2D and 3D Digital Images'. CVGIP: Graph. Models Image Process. 55(1), pp. 20-47

[16] Lohmann, G. (1998): Volumetric Image Analysis (Wiley-Teubner, Chichester, New York)

[17] Matheron, G. (1975): Random Sets and Integral Geometry (Wiley, New York)

[18] Mecke, K. R. (1993): Integralgeometrie in der Statistischen Physik (Harri Deutsch, Frankfurt a. M.)

[19] Mecke, K. R. (2000): 'Additivity, convexity, and beyond: applications of Minkowski functionals in statistical physics'. In: Statistical Physics and Spatial Statistics, edited by K. R. Mecke, D. Stoyan (Springer, Heidelberg), pp. 111-184

[20] Mecke, J., R. G. Schneider, D. Stoyan, W. R. R. Weil (1990): Stochastische Geometrie (Birkhuser, Basel, Boston, Berlin)

[21] Miles, R. (1976): 'Estimating aggregate and overall characterictics from thick sections by transmission microscopy'. J. Microscopy 107, pp. 227-233

[22] Nagel, W., J. Ohser, K. Pischang (2000): 'An integral-geometric approach for the EulerPoincaré characteristic of spatial images'. J. Microsc. 189, pp. 54-62

[23] Ohser, J., F. Mücklich (2000): Statistical Analysis of Materials Structures (J Wiley \& Sons, Chichester, New York)

[24] Ohser, J., W. Nagel (1996): 'The estimation of the Euler-Poincaré characteristic from observations on parallel sections'. J. Microsc. 184, pp. 117-126

[25] Rosenfeld, A., A. Kak (1979): Digital Picture Processing (Academic Press, New York)

[26] Rosenfeld, A., T. Y. Kong, A. Y. Wu (1991): 'Digital surfaces'. CVGIP: Graph. Models Image Process. 53, pp. 305-312

[27] Schneider, R. (1993): Convex Bodies: The Brunn-Minkowski Theory, vol. 44 of Encyclopedia of Mathematics and Its Application (Cambridge University Press, Cambridge)

[28] Schneider, R., W. Weil (2000): Stochastische Geometrie (Teubner, Stuttgart)

[29] Serra, J. (1969): Introduction á la Morphologie Mathématique, (Cahiers du Centre de Morphologie Mathématique, Booklet No. 3, E.N.S.M.P., Paris)

[30] Serra, J. (1982): Image Analysis and Mathematical Morphology, vol. 1 (Academic Press, London) 
[31] Voss, K. (1993): Discrete Images, Objects, and Functions in $Z^{n}$ (Springer-Verlag, New York)

[32] Webster, R. J. (1994): Convexity (Oxford University Press, New York)

[33] Weil, W. (2000): 'Mixed measures and homogeneous Boolean models'. In: Statistical Physics and Spatial Statistics, edited by K. R. Mecke, D. Stoyan (Springer, Heidelberg), pp. $95-110$ 


\author{
Die PDF-Files der folgenden Berichte \\ finden Sie unter: \\ www.itwm.fhg.de/zentral/berichte.html
}

1. D. Hietel, K. Steiner, J. Struckmeier

\section{A Finite - Volume Particle Method for} Compressible Flows

We derive a new class of particle methods for conservation laws, which are based on numerical flux functions to model the interactions between moving particles. The derivation is similar to that of classical Finite-Volume methods; except that the fixed grid structure in the Finite-Volume method is substituted by so-called mass packets of particles. We give some numerical results on a shock wave solution for Burgers equation as well as the well-known one-dimensional shock tube problem. (19 S., 1998)

\section{M. Feldmann, S. Seibold}

\section{Damage Diagnosis of Rotors: Application of Hilbert Transform and Multi-Hypothesis Testing}

In this paper, a combined approach to damage diagnosis of rotors is proposed. The intention is to employ signalbased as well as model-based procedures for an improved detection of size and location of the damage. In a first step, Hilbert transform signal processing techniques allow for a computation of the signal envelope and the instantaneous frequency, so that various types of nonlinearities due to a damage may be identified and classified based on measured response data. In a second step, a multi-hypothesis bank of Kalman Filters is employed for the detection of the size and location of the damage based on the information of the type of damage provided by the results of the Hilbert transform.

Keywords:

Hilbert transform, damage diagnosis, Kalman filtering, non-linear dynamics

(23 S., 1998)

\section{Y. Ben-Haim, S. Seibold}

\section{Robust Reliability of Diagnostic Multi- Hypothesis Algorithms: Application to Rotating Machinery}

Damage diagnosis based on a bank of Kalman filters, each one conditioned on a specific hypothesized system condition, is a well recognized and powerful diagnostic tool. This multi-hypothesis approach can be applied to a wide range of damage conditions. In this paper, we will focus on the diagnosis of cracks in rotating machinery. The question we address is: how to optimize the multihypothesis algorithm with respect to the uncertainty of the spatial form and location of cracks and their resulting dynamic effects. First, we formulate a measure of the reliability of the diagnostic algorithm, and then we discuss modifications of the diagnostic algorithm for the maximization of the reliability. The reliability of a diagnostic algorithm is measured by the amount of uncertainty consistent with no-failure of the diagnosis. Uncertainty is quantitatively represented with convex models. Keywords:

Robust reliability, convex models, Kalman filtering, multihypothesis diagnosis, rotating machinery, crack diagnosis (24 S., 1998)
4. F.-Th. Lentes, N. Siedow

Three-dimensional Radiative Heat Transfer in Glass Cooling Processes

For the numerical simulation of 3D radiative heat transfer in glasses and glass melts, practically applicable mathematical methods are needed to handle such problems optimal using workstation class computers. Since the exact solution would require super-computer capabilities we concentrate on approximate solutions with a high degree of accuracy. The following approaches are studied: 3D diffusion approximations and 3D ray-tracing methods

(23 S., 1998)

\section{A. Klar, R. Wegener}

\section{A hierarchy of models for multilane vehicular traffic Part l: Modeling}

In the present paper multilane models for vehicular traffic are considered. A microscopic multilane model based on reaction thresholds is developed. Based on this model an Enskog like kinetic model is developed. In particular, care is taken to incorporate the correlations between the vehicles. From the kinetic model a fluid dynamic model is derived. The macroscopic coefficients are deduced from the underlying kinetic model. Numerical simulations are presented for all three levels of description in [10]. Moreover, a comparison of the results is given there. (23 S., 1998)

\section{Part II: Numerical and stochastic investigations}

In this paper the work presented in [6] is continued. The present paper contains detailed numerical investigations of the models developed there. A numerical method to treat the kinetic equations obtained in [6] are presented and results of the simulations are shown. Moreover, the stochastic correlation model used in [6] is described and investigated in more detail.

(17 S., 1998)

\section{A. Klar, N. Siedow}

Boundary Layers and Domain Decomposition for Radiative Heat Transfer and Diffusion Equations: Applications to Glass Manufacturing Processes

In this paper domain decomposition methods for radiative transfer problems including conductive heat transfer are treated. The paper focuses on semi-transparent materials, like glass, and the associated conditions at the interface between the materials. Using asymptotic analysis we derive conditions for the coupling of the radiative transfer equations and a diffusion approximation. Several test cases are treated and a problem appearing in glass manufacturing processes is computed. The results clearly show the advantages of a domain decomposition ap proach. Accuracy equivalent to the solution of the global radiative transfer solution is achieved, whereas computation time is strongly reduced.

(24 S., 1998)

\section{I. Choquet}

Heterogeneous catalysis modelling and numerical simulation in rarified gas flows Part I: Coverage locally at equilibrium

A new approach is proposed to model and simulate numerically heterogeneous catalysis in rarefied gas flows. It is developed to satisfy all together the following points: 1) describe the gas phase at the microscopic scale, as required in rarefied flows,

2) describe the wall at the macroscopic scale, to avoid prohibitive computational costs and consider not only crystalline but also amorphous surfaces,

3) reproduce on average macroscopic laws correlated with experimental results and

4) derive analytic models in a systematic and exact way. The problem is stated in the general framework of a non static flow in the vicinity of a catalytic and non porous surface (without aging). It is shown that the exact and systematic resolution method based on the Laplace transform, introduced previously by the author to model collisions in the gas phase, can be extended to the present problem. The proposed approach is applied to the modelling of the Eley-Rideal and Langmuir-Hinshelwood recombinations, assuming that the coverage is locally at equilibrium. The models are developed considering one atomic species and extended to the general case of several atomic species. Numerical calculations show that the models derived in this way reproduce with accuracy behaviors observed experimentally.

(24 S., 1998)

\section{J. Ohser, B. Steinbach, C. Lang}

\section{Efficient Texture Analysis of Binary Images}

A new method of determining some characteristics of binary images is proposed based on a special linear filtering. This technique enables the estimation of the area fraction, the specific line length, and the specific integral of curvature. Furthermore, the specific length of the tota projection is obtained, which gives detailed information about the texture of the image. The influence of lateral and directional resolution depending on the size of the applied filter mask is discussed in detail. The technique includes a method of increasing directional resolution for texture analysis while keeping lateral resolution as high as possible.

(17 S., 1998)

\section{J. Orlik}

\section{Homogenization for viscoelasticity of the} integral type with aging and shrinkage

A multi-phase composite with periodic distributed inclusions with a smooth boundary is considered in this contribution. The composite component materials are supposed to be linear viscoelastic and aging (of the non-convolution integral type, for which the Laplace transform with respect to time is not effectively applicable) and are subjected to isotropic shrinkage. The free shrinkage deformation can be considered as a fictitious temperature deformation in the behavior law. The procedure presented in this paper proposes a way to determine average (effective homogenized) viscoelastic and shrinkage (temperature) composite properties and the homogenized stress-field from known properties of the 
components. This is done by the extension of the asymptotic homogenization technique known for pure elastic non-homogeneous bodies to the non-homogeneous thermo-viscoelasticity of the integral non-convolution type. Up to now, the homogenization theory has not covered viscoelasticity of the integral type.

Sanchez-Palencia (1980), Francfort \& Suquet (1987) (see [2], [9]) have considered homogenization for viscoelasticity of the differential form and only up to the first derivative order. The integral-modeled viscoelasticity is more general then the differential one and includes almost all known differential models. The homogenization procedure is based on the construction of an asymptotic solution with respect to a period of the composite structure. This reduces the original problem to some auxiliary boundary value problems of elasticity and viscoelasticity on the unit periodic cell, of the same type as the original non-homogeneous problem. The existence and uniqueness results for such problems were obtained for kernels satisfying some constrain conditions. This is done by the extension of the Volterra integral operator theory to the Volterra operators with respect to the time, whose 1 kernels are space linear operators for any fixed time variables. Some ideas of such approach were proposed in [11] and [12], where the Volterra operators with kernels depending additionally on parameter were considered. This manuscript delivers results of the same nature for the case of the space-operator kernels.

(20 S., 1998)

\section{J. Mohring}

\section{Helmholtz Resonators with Large Aperture}

The lowest resonant frequency of a cavity resonator is usually approximated by the classical Helmholtz formula. However, if the opening is rather large and the front wal is narrow this formula is no longer valid. Here we present a correction which is of third order in the ratio of the diameters of aperture and cavity. In addition to the high accuracy it allows to estimate the damping due to radiation. The result is found by applying the method of matched asymptotic expansions. The correction contains form factors describing the shapes of opening and cavity. They are computed for a number of standard geometries. Results are compared with numerical computations. (21 S., 1998)

\section{H. W. Hamacher, A. Schöbel \\ On Center Cycles in Grid Graphs}

Finding "good" cycles in graphs is a problem of great interest in graph theory as well as in locational analysis. We show that the center and median problems are NP hard in general graphs. This result holds both for the vari able cardinality case (i.e. all cycles of the graph are considered) and the fixed cardinality case (i.e. only cycles with a given cardinality $p$ are feasible). Hence it is of interest to investigate special cases where the problem is solvable in polynomial time.

In grid graphs, the variable cardinality case is, for instance, trivially solvable if the shape of the cycle can be chosen freely.

If the shape is fixed to be a rectangle one can analyze rectangles in grid graphs with, in sequence, fixed dimension, fixed cardinality, and variable cardinality. In all cases a complete characterization of the optimal cycles and closed form expressions of the optimal objective values are given, yielding polynomial time algorithms for all cases of center rectangle problems.

Finally, it is shown that center cycles can be chosen as rectangles for small cardinalities such that the center cycle problem in grid graphs is in these cases completely solved.

(15 S., 1998)

\section{H. W. Hamacher, K.-H. Küfer}

\section{Inverse radiation therapy planning -} a multiple objective optimisation approach

For some decades radiation therapy has been proved successful in cancer treatment. It is the major task of clinical radiation treatment planning to realize on the one hand a high level dose of radiation in the cancer tissue in order to obtain maximum tumor control. On the other hand it is obvious that it is absolutely necessary to keep in the tissue outside the tumor, particularly in organs at risk, the unavoidable radiation as low as possible. No doubt, these two objectives of treatment planning high level dose in the tumor, low radiation outside the tumor - have a basically contradictory nature. Therefore, it is no surprise that inverse mathematical models with dose distribution bounds tend to be infeasible in most cases. Thus, there is need for approximations compromising between overdosing the organs at risk and underdosing the target volume.

Differing from the currently used time consuming iterative approach, which measures deviation from an ideal (non-achievable) treatment plan using recursively trialand-error weights for the organs of interest, we go a new way trying to avoid a priori weight choices and consider the treatment planning problem as a multiple objective linear programming problem: with each organ of interest, target tissue as well as organs at risk, we associate an objective function measuring the maximal deviation from the prescribed doses.

We build up a data base of relatively few efficient solutions representing and approximating the variety of Pareto solutions of the multiple objective linear programming problem. This data base can be easily scanned by physicians looking for an adequate treatment plan with the aid of an appropriate online tool.

(14 S., 1999)

\section{C. Lang, J. Ohser, R. Hilfer}

\section{On the Analysis of Spatial Binary Images}

This paper deals with the characterization of microscopically heterogeneous, but macroscopically homogeneous spatial structures. A new method is presented which is strictly based on integral-geometric formulae such as Crofton's intersection formulae and Hadwiger's recursive definition of the Euler number. The corresponding algorithms have clear advantages over other techniques. As an example of application we consider the analysis of spatial digital images produced by means of Computer Assisted Tomography.

(20 S., 1999)

\section{M. Junk}

\section{On the Construction of Discrete Equilibrium} Distributions for Kinetic Schemes

A general approach to the construction of discrete equilibrium distributions is presented. Such distribution func tions can be used to set up Kinetic Schemes as well as Lattice Boltzmann methods. The general principles are also applied to the construction of Chapman Enskog distributions which are used in Kinetic Schemes for com- pressible Navier-Stokes equations. (24 S., 1999)

\section{M. Junk, S. V. Raghurame Rao}

\section{A new discrete velocity method for Navier Stokes equations}

The relation between the Lattice Boltzmann Method which has recently become popular, and the Kinetic Schemes, which are routinely used in Computational Fluid Dynamics, is explored. A new discrete velocity mode for the numerical solution of Navier-Stokes equations for incompressible fluid flow is presented by combining both the approaches. The new scheme can be interpreted as a pseudo-compressibility method and, for a particular choice of parameters, this interpretation carries over to the Lattice Boltzmann Method. (20 S., 1999)

\section{H. Neunzert}

\section{Mathematics as a Key to Key Technologies}

The main part of this paper will consist of examples, how mathematics really helps to solve industrial problems; these examples are taken from our Institute for Industrial Mathematics, from research in the Technomathematics group at my university, but also from ECMI groups and a company called TecMath, which originated 10 years ago from my university group and has already a very successful history.

39 S. (vier PDF-Files), 1999)

\section{J. Ohser, K. Sandau}

\section{Considerations about the Estimation of the} Size Distribution in Wicksell's Corpuscle Problem

Wicksell's corpuscle problem deals with the estimation of the size distribution of a population of particles, all having the same shape, using a lower dimensional sampling probe. This problem was originary formulated for particle systems occurring in life sciences but its solution is of actual and increasing interest in materials science. From a mathematical point of view, Wicksell's problem is an inverse problem where the interesting size distribution is the unknown part of a Volterra equation. The problem is often regarded ill-posed, because the structure of the integrand implies unstable numerical solutions. The accuracy of the numerical solutions is considered here using the condition number, which allows to compare different numerical methods with different (equidistant) class sizes and which indicates, as one result, that a finite section thickness of the probe reduces the numerical problems. Furthermore, the relative error of estimation is computed which can be split into two parts. One part consists of the relative discretization error that increases for increasing class size, and the second part is related to the relative statistical error which increases with decreasing class size. For both parts, upper bounds can be given and the sum of them indicates an optimal class width depending on some specific constants.

(18 S., 1999) 
18. E. Carrizosa, H. W. Hamacher, R. Klein, S. Nickel

\section{Solving nonconvex planar location problems by finite dominating sets}

It is well-known that some of the classical location problems with polyhedral gauges can be solved in polynomia time by finding a finite dominating set, i. e. a finite set of candidates guaranteed to contain at least one optimal location.

In this paper it is first established that this result holds for a much larger class of problems than currently considered in the literature. The model for which this result can be proven includes, for instance, location problems with attraction and repulsion, and location-allocation problems. Next, it is shown that the approximation of general gauges by polyhedral ones in the objective function of our general model can be analyzed with regard to the subsequent error in the optimal objective value. For the approx imation problem two different approaches are described, the sandwich procedure and the greedy algorithm. Both of these approaches lead - for fixed epsilon - to polynomial approximation algorithms with accuracy epsilon for solving the general model considered in this paper. Keywords:

Continuous Location, Polyhedral Gauges, Finite Dominating Sets, Approximation, Sandwich Algorithm, Greedy Algorithm

(19 S., 2000)

\section{A. Becker}

\section{A Review on Image Distortion Measures}

Within this paper we review image distortion measures. A distortion measure is a criterion that assigns a "quality number" to an image. We distinguish between mathematical distortion measures and those distortion measures in-cooperating a priori knowledge about the imaging devices (e. g. satellite images), image processing algorithms or the human physiology. We will consider representative examples of different kinds of distortion measures and are going to discuss them. Keywords:

Distortion measure, human visual system (26 S., 2000)

\section{H. W. Hamacher, M. Labbé, S. Nickel} T. Sonneborn

\section{Polyhedral Properties of the Uncapacitated Multiple Allocation Hub Location Problem}

We examine the feasibility polyhedron of the uncapacitated hub location problem (UHL) with multiple allocation, which has applications in the fields of air passenger and cargo transportation, telecommunication and postal delivery services. In particular we determine the dimension and derive some classes of facets of this polyhedron. We develop some general rules about lifting facets from the uncapacitated facility location (UFL) for UHL and projecting facets from UHL to UFL. By applying these rules we get a new class of facets for UHL which dominates the inequalities in the original formulation. Thus we get a new formulation of UHL whose constraints are all facetdefining. We show its superior computational performance by benchmarking it on a well known data set Keywords:

integer programming, hub location, facility location, valid inequalities, facets, branch and cut

(21 S., 2000)

\section{H. W. Hamacher, A. Schöbel \\ Design of Zone Tariff Systems in Public Transportation}

Given a public transportation system represented by its stops and direct connections between stops, we consider two problems dealing with the prices for the customers: The fare problem in which subsets of stops are already aggregated to zones and "good" tariffs have to be found in the existing zone system. Closed form solutions for the fare problem are presented for three objective functions. In the zone problem the design of the zones is part of the problem. This problem is NP hard and we therefore propose three heuristics which prove to be very successful in the redesign of one of Germany's transportation systems. (30 S., 2001)

\section{D. Hietel, M. Junk, R. Keck, D. Teleaga: \\ The Finite-Volume-Particle Method for Conservation Laws}

In the Finite-Volume-Particle Method (FVPM), the weak formulation of a hyperbolic conservation law is discretized by restricting it to a discrete set of test functions. In contrast to the usual Finite-Volume approach, the test functions are not taken as characteristic functions of the control volumes in a spatial grid, but are chosen from a partition of unity with smooth and overlapping partition functions (the particles), which can even move along prescribed velocity fields. The information exchange between particles is based on standard numerical flux functions. Geometrical information, similar to the surface area of the cell faces in the Finite-Volume Method and the corresponding normal directions are given as integral quantities of the partition functions.

After a brief derivation of the Finite-Volume-Particle Method, this work focuses on the role of the geometric coefficients in the scheme.

(16 S., 2001)

23. T. Bender, H. Hennes, J. Kalcsics,

M. T. Melo, S. Nickel

\section{Location Software and Interface with GIS and Supply Chain Management}

The objective of this paper is to bridge the gap between location theory and practice. To meet this objective focus is given to the development of software capable of addressing the different needs of a wide group of users. There is a very active community on location theory encompassing many research fields such as operations research, computer science, mathematics, engineering, geography, economics and marketing. As a result, people working on facility location problems have a very diverse background and also different needs regarding the software to solve these problems. For those interested in non-commercial applications (e. g. students and researchers), the library of location algorithms (LoLA can be of considerable assistance. LoLA contains a collection of efficient algorithms for solving planar, network and discrete facility location problems. In this paper, a detailed description of the functionality of LoLA is presented. In the fields of geography and marketing, for instance, solving facility location problems requires using large amounts of demographic data. Hence, members of these groups (e. g. urban planners and sales managers) often work with geographical information too $\mathrm{s}$. To address the specific needs of these users, LoLA was inked to a geo- graphical information system (GIS) and the details of the combined functionality are described in the paper. Finally, there is a wide group of practitioners who need to solve large problems and require special purpose software with a good data interface. Many of such users can be found for example, in the area of supply chain management (SCM). Logistics activities involved in strategic SCM include, among others, facility location planning. In this paper, the development of a commercial location software tool is also described. The too is embedded in the Advanced Planner and Optimizer SCM software developed by SAP AG, Walldorf, Germany. The paper ends with some conclusions and an outlook to future activities.

Keywords:

facility location, software development, geographical information systems, supply chain management. (48 S., 2001)

\section{H. W. Hamacher, S. A. Tjandra Mathematical Modelling of Evacuation Problems: A State of Art}

This paper details models and algorithms which can be applied to evacuation problems. While it concentrates on building evacuation many of the results are applicable also to regional evacuation. All models consider the time as main parameter, where the travel time between components of the building is part of the input and the overall evacuation time is the output. The paper distinguishes between macroscopic and microscopic evacuation models both of which are able to capture the evacuees' movement over time.

Macroscopic models are mainly used to produce good lower bounds for the evacuation time and do not consider any individual behavior during the emergency situation. These bounds can be used to analyze existing buildings or help in the design phase of planning a building Macroscopic approaches which are based on dynamic network flow models (minimum cost dynamic flow, maximum dynamic flow, universal maximum flow, quickest path and quickest flow) are described. A special feature of the presented approach is the fact, that travel times of evacuees are not restricted to be constant, but may be density dependent. Using multicriteria optimization priority regions and blockage due to fire or smoke may be considered. It is shown how the modelling can be done using time parameter either as discrete or continuous parameter

Microscopic models are able to model the individual evacuee's characteristics and the interaction among evacuees which influence their movement. Due to the corresponding huge amount of data one uses simulation approaches. Some probabilistic laws for individual evacuee's movement are presented. Moreover ideas to model the evacuee's movement using cellular automata (CA) and resulting software are presented.

In this paper we will focus on macroscopic models and only summarize some of the results of the microscopic approach. While most of the results are applicable to general evacuation situations, we concentrate on building evacuation.

(44 S., 2001) 
25. J. Kuhnert, S. Tiwari

\section{Grid free method for solving the Poisson equation}

A Grid free method for solving the Poisson equation is presented. This is an iterative method. The method is based on the weighted least squares approximation in which the Poisson equation is enforced to be satisfied in every iterations. The boundary conditions can also be enforced in the iteration process. This is a local approximation procedure. The Dirichlet, Neumann and mixed boundary value problems on a unit square are presented and the analytical solutions are compared with the exact solutions. Both solutions matched perfectly. Keywords:

Poisson equation, Least squares method, Grid free method (19 S., 2001)

26. T. Götz, H. Rave, D. Reinel-Bitzer, K. Steiner, H. Tiemeier

\section{Simulation of the fiber spinning process}

To simulate the influence of process parameters to the melt spinning process a fiber model is used and coupled with CFD calculations of the quench air flow. In the fiber model energy, momentum and mass balance are solved for the polymer mass flow. To calculate the quench air the Lattice Boltzmann method is used. Simulations and experiments for different process parameters and hole configurations are compared and show a good agreement.

Keywords:

Melt spinning, fiber model, Lattice Boltzmann, CFD (19 S., 2001)

\section{A. Zemitis}

\section{On interaction of a liquid film with an obstacle}

In this paper mathematical models for liquid films generated by impinging jets are discussed. Attention is stressed to the interaction of the liquid film with some obstacle. S. G. Taylor [Proc. R. Soc. London Ser. A 253, 313 (1959)] found that the liquid film generated by impinging jets is very sensitive to properties of the wire which was used as an obstacle. The aim of this presentation is to propose a modification of the Taylor's model, which allows to simulate the film shape in cases, when the angle between jets is different from $180^{\circ}$. Numerical results obtained by discussed models give two different shapes of the liquid film similar as in Taylors experiments. These two shapes depend on the regime: either droplets are produced close to the obstacle or not. The difference between two regimes becomes larger if the angle between jets decreases. Existence of such two regimes can be very essential for some applications of impinging jets, if the generated liquid film can have a contact with obstacles. Keywords:

impinging jets, liquid film, models, numerical solution, shape

(22 S., 2001)

\section{I. Ginzburg, K. Steiner}

\section{Free surface lattice-Boltzmann method to model the filling of expanding cavities by Bingham Fluids}

The filling process of viscoplastic metal alloys and plastics in expanding cavities is modelled using the lattice Boltzmann method in two and three dimensions. These mod els combine the regularized Bingham model for viscoplastic with a free-interface algorithm. The latter is based on a modified immiscible lattice Boltzmann model in which one species is the fluid and the other one is considered as vacuum. The boundary conditions at the curved liquid-vacuum interface are met without any geometrical front reconstruction from a first-order ChapmanEnskog expansion. The numerical results obtained with these models are found in good agreement with available theoretical and numerical analysis. Keywords:

Generalized LBE, free-surface phenomena, interface boundary conditions, filling processes, Bingham viscoplastic model, regularized models (22 S., 2001)

\section{H. Neunzert}

"Denn nichts ist für den Menschen als Menschen etwas wert, was er nicht mit Leidenschaft tun kann"

Vortrag anlässlich der Verleihung des Akademiepreises des Landes Rheinland-Pfalz am

\subsubsection{1}

Was macht einen guten Hochschullehrer aus? Auf diese Frage gibt es sicher viele verschiedene, fachbezogene Antworten, aber auch ein paar allgemeine Gesichtspunkte: es bedarf der »Leidenschaft « für die Forschung (Max Weber), aus der dann auch die Begeisterung für die Lehre erwächst. Forschung und Lehre gehören zusammen, um die Wissenschaft als lebendiges Tun vermitteln zu können. Der Vortrag gibt Beispiele dafür, wie in angewandter Mathematik Forschungsaufgaben aus praktischen Alltagsproblemstellungen erwachsen, die in die Lehre auf verschiedenen Stufen (Gymnasium bis Graduiertenkolleg) einfließen; er leitet damit auch zu einem aktuellen Forschungsgebiet, der Mehrskalenanalyse mit ihren vielfältigen Anwendungen in Bildverarbeitung Materialentwicklung und Strömungsmechanik über, was aber nur kurz gestreift wird. Mathematik erscheint hier als eine moderne Schlüsseltechnologie, die aber auch enge Beziehungen zu den Geistes- und Sozialwissenschaften hat.

Keywords:

Lehre, Forschung, angewandte Mathematik, Mehrskalenanalyse, Strömungsmechanik

(18 S., 2001)

30. J. Kuhnert, S. Tiwari

Finite pointset method based on the projection method for simulations of the incom pressible Navier-Stokes equations

A Lagrangian particle scheme is applied to the projection method for the incompressible Navier-Stokes equations. The approximation of spatial derivatives is obtained by the weighted least squares method. The pressure Poisson equation is solved by a local iterative procedure with the help of the least squares method. Numerical tests are performed for two dimensional cases. The Couette flow Poiseuelle flow, decaying shear flow and the driven cavity flow are presented. The numerical solutions are obtained for stationary as well as instationary cases and are compared with the analytical solutions for channel flows. Finally, the driven cavity in a unit square is considered and the stationary solution obtained from this scheme is compared with that from the finite element method. Keywords:

Incompressible Navier-Stokes equations, Meshfree method, Projection method, Particle scheme, Least squares approximation

AMS subject classification:

76D05, 76M28

(25 S., 2001)

\section{R. Korn, M. Krekel Optimal Portfolios with Fixed Consumption or Income Streams}

We consider some portfolio optimisation problems where either the investor has a desire for an a priori specified consumption stream or/and follows a deterministic pay in scheme while also trying to maximize expected utility from final wealth. We derive explicit closed form solutions for continuous and discrete monetary streams. The mathematical method used is classical stochastic control theory.

Keywords:

Portfolio optimisation, stochastic control, HJB equation, discretisation of control problems.

(23 S., 2002)

\section{M. Krekel}

\section{Optimal portfolios with a loan dependent credit spread}

If an investor borrows money he generally has to pay higher interest rates than he would have received, if he had put his funds on a savings account. The classical model of continuous time portfolio optimisation ignores this effect. Since there is obviously a connection between the default probability and the total percentage of wealth, which the investor is in debt, we study portfolio optimisation with a control dependent interest rate. Assuming a logarithmic and a power utility function, respectively, we prove explicit formulae of the optimal control.

Keywords:

Portfolio optimisation, stochastic control, HJB equation, credit spread, log utility, power utility, non-linear wealth dynamics

(25 S., 2002)

\section{J. Ohser, W. Nagel, K. Schladitz}

The Euler number of discretized sets - on the choice of adjacency in homogeneous lattices

Two approaches for determining the Euler-Poincaré characteristic of a set observed on lattice points are considered in the context of image analysis \{ the integral geometric and the polyhedral approach. Information about the set is assumed to be available on lattice points only. In order to retain properties of the Euler number and to provide a good approximation of the true Euler number of the original set in the Euclidean space, the appropriate choice of adjacency in the lattice for the set and its background is crucial. Adjacencies are defined using tessellations of the whole space into polyhedrons. In $\mathrm{R} 3$, two new 14 adjacencies are introduced additionally to the 
well known 6 and 26 adjacencies. For the Euler number of a set and its complement, a consistency relation holds. Each of the pairs of adjacencies $(14: 1 ; 14: 1),(14: 2 ; 14: 2)$ $(6 ; 26)$, and $(26 ; 6)$ is shown to be a pair of complementary adjacencies with respect to this relation. That is, the approximations of the Euler numbers are consistent if the set and its background (complement) are equipped with this pair of adjacencies. Furthermore, sufficient conditions for the correctness of the approximations of the Euler number are given. The analysis of selected microstructures and a simulation study illustrate how the estimated Euler number depends on the chosen adjacency. It also shows that there is not a uniquely best pair of adjacencies with respect to the estimation of the Euler number of a set in Euclidean space.

Keywords: image analysis, Euler number, neighborhod relationships, cuboidal lattice

(32 S., 2002 